GRASAS Y ACEITES 65 (2)

April-June 2014, e028

ISSN-L: 0017-3495

doi: http://dx.doi.org/10.3989/gya.110913

\title{
La Antracnosis del olivo y su efecto en la calidad del aceite
}

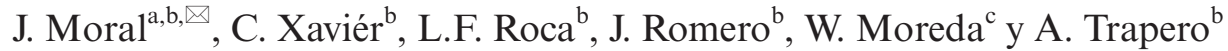

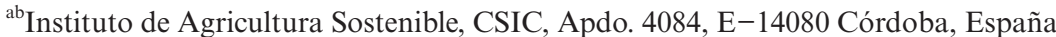 \\ ${ }^{\text {b}}$ Departamento de Agronomía, ETSIAM, Universidad de Córdoba, Campus de Rabanales, Edif. C4, 14071 Córdoba, España \\ ${ }^{\circ}$ Instituto de la Grasa, CSIC, Avda. Padre García Tejero, 4, Sevilla, España \\ Corresponding author: jmoral@ias.csic.es
}

Submitted: 7 November 2013; Accepted: 17 January 2014

SUMMARY: Olive Anthracnose and its effect on oil quality. Olive (Olea europaea subsp. europaea var. europaea) is one of the first domesticated and cultivated trees that is widely distributed in the Mediterranean regions. The Anthracnose, caused by the two complex fungal species Colletotrichum acutatum and C. gloeosporioides, is the most important disease adversely affecting the olive oil quality. Even so, the effect of Anthracnose on oil quality is largely unknown and many questions remain unanswered. This offers a unique opportunity to study how Colletotrichum species, cultivars, infection type (latent or visible) and severity, and other factors that may affect different parameters of oil quality, such us acidity, peroxide value, K232, K270, phenolic compounds, or alkyl esters. This review focuses on the current knowledge of the biology, epidemiology, and management of Anthracnose and its effect on olive oil quality.

KEYWORDS: Oil quality; Olive; Soapy rot; Virgin oil

RESUMEN: El olivo (Olea europaea subsp. europaea var. europaea) es uno de los primeros cultivos leñosos domesticados. Actualmente el olivo se encuentra ampliamente distribuido por todas las regiones de clima mediterráneo. La Antracnosis, causada por las especies complejas Colletotrichum acutatum y C. gloeosporioides, es la enfermedad del olivo que más afecta a la calidad del aceite. Aun así, el efecto de la Antracnosis en la calidad del aceite es ampliamente desconocido. Por lo que creemos esencial que se afronten estudios encaminados a dilucidar el efecto de las especies del patógeno, el cultivar de olivo y el tipo (latente o visible) y severidad de las infecciones de la aceituna en los distintos parámetros de calidad del aceite como la acidez, índice de peróxidos, K232, K270, compuestos fenólicos o ésteres alquílicos. Esta revisión presenta los conocimientos actuales sobre la biología, epidemiología, control, y efecto en la calidad del aceite de la Antracnosis del olivo.

PALABRAS CLAVE: Aceite de oliva virgen; Aceituna jabonosa; Calidad de aceite; Olivo

Citation/Cómo citar este artículo: Moral J, Xaviér C, Roca LF, Romero J, Moreda W, Trapero A. 2014. La Antracnosis del olivo y su efecto en la calidad del aceite. Grasas Aceites 65 (2): e028. doi: http://dx.doi.org/10.3989/gya.110913.

Copyright: (c) 2014 CSIC. This is an open-access article distributed under the terms of the Creative Commons Attribution-Non Commercial (by-nc) Spain 3.0 Licence. 


\section{EL OLIVO}

El olivo (Olea europaea subsp. europaea var. europaea) es miembro de la familia Oleaceae junto a unas 600 especies entre las que se encuentran el aligustre (Ligustrum), fresno (Fraxinus) y jazmín (Jasminun). Dentro de la especie O. europea se insertan distintas subespecies como laperrinei, cuspidata, guanchita, marroccana y cersiformis (Green, 2002), aunque su correcta ubicación filogenética está en continuo cambio. El olivo es una especie que de forma natural ocupa las orillas del Mar Mediterráneo, aunque de la mano del hombre ha colonizado zonas de clima mediterráneo del hemisferio sur como Argentina, Australia, Chile y Sudáfrica (Zohary, 1994; FAO, 2012).

El acebuche u olivo silvestre (Olea europaea subsp. europaea var. sylvestris), es la variedad botánica más próxima al olivo cultivado y de la cual procede. En general, se acepta que el olivo se domesticó mediante selección automática de los individuos más sobresalientes de acebuche en el Oriente Medio durante la Edad del Bronce, hace unos 6000-5500 años (Zohary y Spiegel-Roy, 1975). Posteriormente, estas variedades primigenias fueron propagadas vegetativamente y distribuidas por ambas orillas del Mediterráneo por los fenicios y posteriores civilizaciones (Rallo, 2005). A pesar de ello, no se descarta que hubiesen procesos de domesticación secundarios en otros puntos alejados del Medio Oriente como España o el Magreb, aunque estas zonas están consideras como centros de diversificación y no domesticación (Besnard et al., 2013). Actualmente, se calcula que existen 2.500 variedades de olivo distribuidas localmente (Caballero et al., 2006) aunque unas pocas han colonizando una amplio rango de zonas agroclimáticas (Bronzini de Caraffa et al., 2002).

El olivo es una de las fuentes más importante de aceite vegetal para el consumo humano. Varios estudios realizados entre los años 1950-1970 por el Dr. Keys y colaboradores asociaron la dieta mediterránea, y en particular el consumo de aceite de oliva, con el escaso número de fallecimientos por enfermedades cardiovasculares en la isla griega de Creta (38/10000 habitantes) comparado con otros países como Estados Unidos (773/10000 hab). Esta diferencia se acentuaba aún más en el caso de Finlandia (1202/10000 hab) cuyos habitantes tenían un nivel de consumo de grasas similar al de los cretenses aunque mayoritariamente de origen animal (Keys et al., 1952; Keys, 1970; Matalas et al., 2001). El aceite de oliva ha sido declarado como un "alimento medicinal" por la Administración Americana de Alimentos y Medicamentos (FDA) debido a su efecto protector contra las enfermedades cardiovasculares uniéndose al grupo de alimentos medicinales junto a las avellanas y los ácidos grasos omega-3 (www.fda.gov).
Entre las enfermedades aéreas que afectan al olivo destacan la Tuberculosis, causada por la bacteria Pseudomonas savastanoi pv. savastanoi; y sobre todo las enfermedades fúngicas Repilo, causada por Spilocaea oleaginea; Emplomado, causada por Pseudocercospora cladosporioides; y la Antracnosis o Aceituna jabonosa, causada por varias especies del género Colletotrichum. Mundialmente, la Antracnosis es la enfermedad más importante de la aceituna (Andrés, 1991; Trapero y Blanco, 2008; Moral et al., 2009c; Cacciola et al., 2012) y, sin duda alguna, la enfermedad que causa un mayor deterioro de la calidad del aceite (García, 1995; 1998; García et al., 1997a; Iannotta et al., 1999; Mincione et al., 2004; Carvalho et al., 2008).

El género Colletotrichum está compuesto por unas 600 especies que afectan a más de 3000 especies de plantas silvestres y cultivadas (Farr y Rossman, 2013). Entre ellas, numerosos cultivos leñosos de zonas tropicales, subtropicales y templadas como almendro (Prunus dulcis), arándano (Vaccinium spp.), cítricos (Citrus spp.), mango (Magnifera indica), melocotonero (Prunus persica) $\mathrm{y}$, frutos con alto contenido lipídico como los del aguacate (Persea americana) (Prusky et al., 2000).

Este trabajo pretende ayudar al sector oleícola a mejorar el conocimiento y control de la enfermedad del olivo que perjudica más seriamente a la calidad organoléptica del aceite. Además, actualiza el conocimiento científico de la enfermedad y establece futuras líneas de colaboración entre especialistas en calidad de aceite y fitopatólogos.

\section{HISTORIA, IMPORTANCIA Y DISTRIBUCIÓN DE LA ANTRACNOSIS}

La Antracnosis fue descrita científicamente por primera vez en Portugal por J. V. d'Almeida quien publicó un breve descripción de la enfermedad y del agente causal (Almeida, 1899). Posteriormente, La Antracnosis ha sido descrita en la mayoría de los países oleícolas del mundo de ambos hemisferios (Tabla 1).

En diversos tratados clásicos de agricultura, como los libros de Columela (siglo I), Plinio (siglo I) y Alonso de Herrera (siglo XVI), se menciona la necesidad de adelantar la recolección en los años lluviosos, reducir el atrojado y eliminar las aceitunas que estén en mal estado para obtener aceite de buena calidad. Aunque, es el agrónomo sevillano Bủ'l-Jayr (siglo XI) quien hace alusión clara a la Aceituna Jabonosa al recomendar procesos especiales de elaboración de aceite "si las aceitunas enferman y se debilitan hasta convertirse en una especie de jabón".

En Italia, es probable que la Antracnosis fuera introducida en el sur desde Albania o Grecia durante la Segunda Guerra Mundial originando gravísimas epidemias durante la década de 1950-60 que afectaron a unas 5000 ha en la región de Apulia (Ciccarone, 
TABla 1. Países en los que ha sido descrita la Antracnosis del Olivo

\begin{tabular}{lll}
\hline País & Año & \multicolumn{1}{c}{ Fuente } \\
\hline Portugal & 1899 & Almedia, 1899 \\
Francia & 1911 & Citado por Bompeix et al., 1998 \\
España & 1914 & González-Fragoso, 1914 \\
Rusia & 1929 & Citado por Farr y Rossman, 2013 \\
Uruguay & 1932 & Citado por Farr y Rossman, 2013 \\
Grecia & 1934 & Biraghi, 1934 \\
Japón & 1935 & Hemi y Murata, 1935 \\
EE.UU & 1942 & Pontis y Hansen, 1942 \\
Brasil & 1943 & Farr y Rossman, 2013 \\
Argentina & 1943 & Citado por Fernández, 1973 \\
Italia & 1950 & Ciccarone, 1950 \\
Sudáfrica & 1956 & Gorter, 1956 \\
Australia & 1962 & Sergeeva et al., 2008a \\
China & 1986 & Margarita et al., 1986 \\
India & 1990 & Sharma y Kaul, 1990 \\
Yugoslavia (antigua) & 1999 & Vucinic et al., 1999 \\
Montenegro & 2002 & Latinovic y Vucinic Z, 2002 \\
Ucrania & 2004 & Citado por Farr y Rossman, 2013 \\
Túnez & 2010 & Rhouma et al., 2010 \\
\hline
\end{tabular}

1950; Graniti y Laviola, 1981). Posteriormente, la enfermedad se extendió en toda la región llegando a afectar a unas 40000 ha detectándose en las islas de Cerdeña y Sicilia (Ciccarone, 1950; Saponaro, 1953; Martelli, 1959, 1960; Graniti et al., 1993). Durante estas epidemias, el patógeno afectó entre el 10 y $65 \%$ de las aceitunas de diversas zonas de la Apulia causando importantes defoliaciones en el cv. Cellina di Nardò (Martelli, 1959; 1960). A partir de 1960 se observó una mengua de la enfermedad que se ha atribuido a la disminución de las precipitaciones, al incremento de tratamientos cúpricos y a un cambio en la virulencia del hongo, al mezclarse las poblaciones patogénicas de olivo con otras locales menos virulentas pero mejor adaptadas (Graniti et al., 1993).

En Portugal, se han descrito varias epidemias severas asociadas al cultivar dominante del país 'Galega vulgar'. A finales de los años 60, las pérdidas debidas a la Antracnosis superaban los 3 millones de dólares en este país (Coutinho, 1968). Años más tarde, Azevedo (1976), calculó que en zonas húmedas de este país el patógeno causaba unas pérdidas próximas al 50\% de la producción de aceite. En estudios realizados entre 2004-2006, Talhinhas et al. (2009) describen una prevalencia de la enfermedad entre el 65 y $100 \%$ y una incidencia del $22 \%$ en los años más secos (2004 y 2005), y del 85\% en el año más lluvioso (2006), en los olivares de la región del Algarve.

En España, la Antracnosis ocupa el $5^{\circ}$ lugar en importancia entre las plagas y enfermedades del olivo, produciendo unas pérdidas medias anuales del
$2.6 \%$ de la cosecha, aunque éstas alcanzan un $40 \%$ en las zonas húmedas del sur y noreste peninsular donde predominan cultivares susceptibles y la enfermedad es endémica (Andrés, 1991; García, 1998; Trapero y Blanco, 2008). Recientemente, hemos estimado que la pérdidas alcanzan los 75.2 millones de euros anuales en España (Moral et al., 2009c). En Andalucía, cabe destacar que el cv. Picual, predominante en la principal provincia oleícola, Jaén, muestra un elevado nivel de resistencia por lo que las epidemias están asociadas a zonas productoras dominadas por los cvs. Hojiblanca, Lechín de Sevilla o Picudo. Aunque carecemos de información precisa, no se registraron epidemias severas de Antracnosis en esta región durante el periodo 1970-1995 coincidiendo con una menor pluviometría. En 1997, el otoño especialmente cálido y lluvioso propició una grave epidemia de esta enfermedad que, en el sur de Córdoba y norte de Málaga, afectó a la totalidad de las aceitunas con la consiguiente pérdida de producción y de calidad de la cosecha (Trapero y Blanco, 2008). Por último, en la campaña oleícola 2012/13, condicionada por la elevada pluviometría y las temperaturas suaves, el patógeno ha afectado gravemente a olivares de las provincias de Córdoba, Málaga y Sevilla.

\section{SINTOMATOLOGÍA}

Los síntomas más característicos de la Antracnosis se manifiestan en las aceitunas maduras aunque pueden observarse también en los frutos verdes 
dependiendo de la resistencia del cultivar, presión de inóculo y condiciones ambientales. Las aceitunas afectadas presentan manchas deprimidas de color ocre o pardo, que crecen y pueden llegar a coalescer, dando lugar a su podredumbre parcial o total. Los ataques se producen en cualquier parte de la aceituna, aunque suelen ser más frecuentes en su zona apical o basal (Martelli, 1960; Mateo-Sagasta, 1968). En aceitunas sin síntomas externos pueden observarse un deterioro del mesocarpo alrededor del hueso que adquiere una tonalidad parda (Agosteo et al., 2005).

Cuando el tiempo es húmedo, sobre las lesiones aparecen los órganos fructíferos asexuales del hongo (conidiomas acervulares o acérvulos), que emiten una sustancia gelatinosa rosa-anaranjada que contiene gran cantidad de esporas (conidios) y que le da el nombre de Aceituna jabonosa (Mateo-Sagasta, 1968; Moral y Trapero, 2009b; Figura 1a). Si baja la humedad y la temperatura se incrementa, las aceitunas afectadas se momifican (Mateo-Sagasta, 1968; Graniti et al., 1993; Figura 1b). El patógeno coloniza el pedúnculo desde el fruto infectado causando su caída (Oliveira et al., 2005) aunque algunas momias pueden quedar en la copa de los árboles entre campañas. El patógeno también puede colonizar las semillas de la aceituna a través del canal peduncular del hueso causando su podredumbre o afectando a la germinación y desarrollo de las plántulas que, en ocasiones, pueden morir (damping-off) (Moral et al., 2009a).

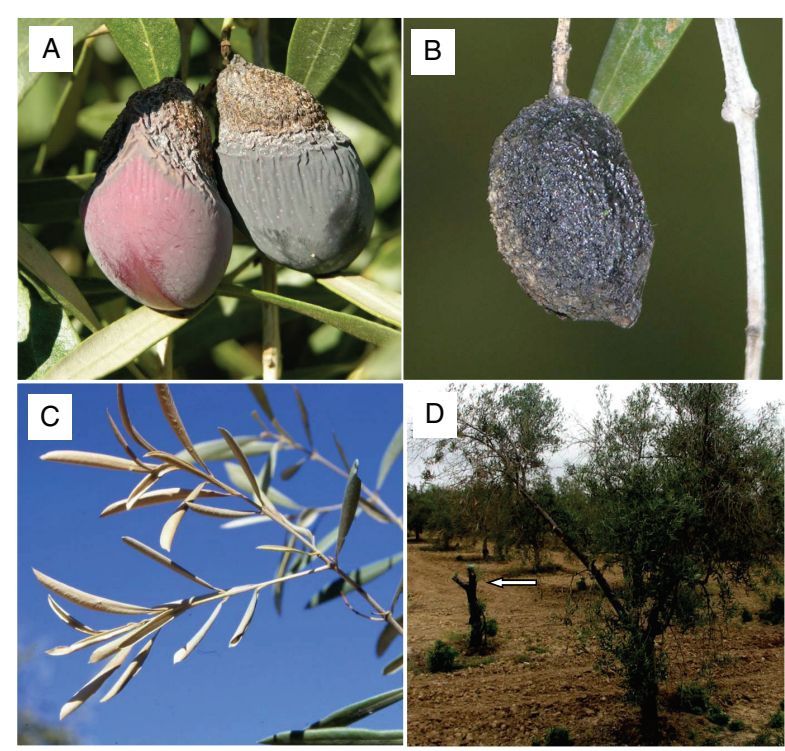

Figura 1. Síntomas de la Antracnosis del olivo causada por Colletotrichum spp. (A) Aceitunas con podredumbre en el área peduncular. (B) Aceituna momificada. (C) Desecación y muerte de ramos fructíferos. (D) Olivo severamente afectado de muerte de ramas junto a un olivo completamente podado (flecha) debido a la enfermedad.
El segundo síndrome asociado a la Antracnosis es la muerte de ramas. Inicialmente, las hojas muestran zonas necróticas que pueden avanzar hasta afectar a su totalidad. Las hojas marchitas terminan por abarquillarse y quedan adheridas a las ramitas dando lugar a un puntisecado generalizado que puede progresar hasta afectar a ramas principales (Martelli, 1960; Zachos y Makris, 1963; Azevedo, 1976; Moral et al., 2009c; Figura 1c). Es frecuente observar árboles que han sido severamente afectados por este segundo síndrome y sometidos a una poda intensa buscando la formación de una nueva copa sana (Figura 1d).

Este segundo síndrome se ha asociado a la producción de toxinas del patógeno en la aceitunas afectadas y que se movilizan al resto del árbol (Moral et al., 2009c). En España, el patógeno puede ser aislado de hojas infectadas aunque no llega a desarrollar acérvulos en condiciones de campo (Moral et al., 2009c) y sólo lo hace cuando las hojas están sometidas a elevada humedad durante varias semanas en condiciones artificiales. Por el contrario, acérvulos en hojas sí se han observado en condiciones naturales en Australia (Sergeeva et al., 2008b), Grecia (Zachos y Makris, 1963) e Italia (Martelli, 1961). El patógeno también causa infecciones de inflorescencias y frutos jóvenes. Estos ataques tienen escasa o nula importancia sobre la cosecha y sólo se han registrado en Sudáfrica (Gorter, 1956), España (Moral et al., 2009c) y Australia (Sergeeva et al., 2008a).

\section{ETIOLOGÍA}

El hongo causante de la Antracnosis fue descrito por Almeida en 1899 con el nombre de Gloeosporium olivarum Alm. Estudios posteriores confirmaron que esta especie era indistinguible de otras especies de Gloeosporium siendo reclasificadas por Von Arx en la especie compleja Colletotrichum gloeosporioides (Penz.) Penz. et Sacc. (Arx, 1970). El género Colletotrichum (teleomorfo Glomerella), contiene aproximadamente 900 especies (Sutton, 1980). En la clasificación tradicional de los hongos, Colletotrichum se encuadra en la división Eumycota, subdivisión Deuteromycotina, clase Coelomycetes y orden Melanconiales (Ainsworth, 1973). Si se clasifica éste atendiendo a su teleomorfo Glomerella, se inserta en el subreino Dikarya, phyllum Ascomycota, subphyllum Pezizomycotina, clase Sordariomycetes y orden Phyllachorales (Hibbet et al., 2007).

En 1986 se detectó la especie C. acutatum Simmonds ex Simmonds atacando al olivo en China (Margarita et al., 1986) y cuya principal característica distintiva era poseer una mayor proporción de conidios fusiformes y estrechos. Posteriormente, esta especie se describió afectando a olivos en India (Mugnai et al., 1993), España (Martín y García, 1999), Italia (Agosteo et al., 2000) y Portugal 
(Talhinhas et al., 2005). La presencia de C. acutatum en estas zonas plantea la cuestión sobre si se trata de una introducción reciente, o bien si han existido diferentes variantes o especies del patógeno bajo la denominación común de $G$ olivarum o C. gloeosporioides.

El incremento del potencial discriminante de las técnicas moleculares ha permitido diferenciar grupos genéticamente homogéneos dentro de las especies originales $C$. acutatum sensu lato (s.l.) y $C$. gloeosporioides s.l. Posteriormente, estos grupos han sido reclasificados como especies nuevas aceptando que las especies de hongos están constituidas por grupos filogenéticamente próximos debido a que las formas intermedias ("híbridos") tienden a desaparecer por una menor adaptación. Tras diversas reclasificaciones, análisis moleculares recientes utilizando varias regiones del ADN han permitido discernir 31 y 22 especies pertenecientes a los complejos $C$. acutatum s.l y $C$. gloeosporioides s.l., respectivamente (Damm et al., 2012; Weir et al., 2012).

De las 31 especies del complejo C. acutatum s.l., seis han sido asociadas con la Antracnosis del olivo: C. acutatum sensu stricto (s.s), C. fiorinae, C. godetiae (=C. clavatum), C. nymphaeae, C. rhombiforme y $C$. simmondsii (Damm et al., 2012). La importancia de estas especies en olivo varía sustancialmente con la región olivarera, destacando $C$. godetiae en Andalucía (Moral et al., 2012) y en Italia (Faedda et al., 2011) y C. nymphaeae en Portugal (Talhinhas et al. 2005) y C. acutatum s.s. en Australia y Sudáfrica (Damm et al., 2012). Aunque en Portugal la especie C. nymphaeae es dominante y la única que se encuentra en muchos olivares, es frecuente encontrar esta especie junto a las restantes especies descritas (Talhinhas et al., 2009). En Andalucía, la especie mayoritaria es $C$. godetiae aunque hemos detectado algunos aislados de $C$. simmondsii en la provincia de Huelva. La especie $C$. godetiae fue identificada en Italia como $C$. clavatum, debido a la morfología claviforme de los conidios (Faedda et al., 2011), pero C. godetiae es el nombre actualmente aceptado por los micólogos (Damm et al., 2012).

De las 22 especies del complejo C. gloeosporioides s. l., seis han sido asociadas con la Antracnosis del olivo: C. aenigma, C. gloeoporioides s.s., C. kahawe sbp. ciggaro, $C$. quenslandium, $C$. siamense y $C$. theobromicola (Schena et al., 2013). La importancia de estas especies en olivar es poco conocida al haberse descritas recientemente. El complejo C. gloeosporioides s.l. está ampliamente distribuido en todas las regiones olivareras, pero su virulencia en aceituna parece menor que la de C. acutatum (Martín et al., 2002), habiéndose postulado como un patógeno secundario (Talhinhas et al., 2009). No obstante, se han observado ataque severos en campo por $C$. gloeosporioides s.l. y los estudios de Schena et al. (2013) han puesto de manifiesto que algunas especies, como C. gloeosporioides S.S. y C. theobromicola, resultan altamente virulentas incluso en aceitunas verdes. En este estudio se identificó también la especie $C$. karstii, del complejo $C$. boninense s.l., como patógena de aceitunas aunque de escasa virulencia.

En ninguno de los estudios donde se aborda la influencia de la Antracnosis en la calidad del aceite de oliva se especifica la especie concreta de Colletotrichum involucrada (García et al., 1997a; García, 1998; Carvalho et al., 2008; Iannotta et al., 1999), de ahí que la influencia de factor especie sea desconocida.

\section{CICLO BIOLÓGICO Y EPIDEMIOLOGÍA}

La principal fuente de inóculo de la Antracnosis son las aceitunas momificadas que quedan en la copa del árbol (Mateo-Sagasta, 1968; Graniti et al., 1993; Moral y Trapero, 2012). Durante el otoño, el patógeno produce una enorme cantidad de conidios (>20 mil conidios/momia) en éstas aunque varía con el cultivar y las condiciones ambientales, siendo las óptimas $20-25^{\circ} \mathrm{C}$ y $96 \mathrm{~h}$ de humectación (Moral y Trapero, 2012). Los conidios son dispersados a corta distancia por las salpicaduras de las gotas lluvia de ahí la distribución en focos de la enfermedad. La dispersión de inóculo a larga distancia se debe a la distribución de plantones infectados o contaminados (Cacciola et al., 2012; Moral et al., 2012) y a la posible dispersión por la mosca del olivo (Agosteo et al., 2006) o la maquinaria de recolección. El teleomorfo del patógeno, cuyas ascosporas podrían dispersarse por el viento a larga distancia, no se ha detectado en condiciones de campo (Cacciola et al., 1996).

Los frutos momificados que caen al suelo, y no se recolectan, se degradan debido a microorganismo secundarios o son enterrados, por lo que participan escasamente en la generación de inóculo si son comparados con las momias aéreas (Graniti et al., 1993; Moral y Trapero, 2012). En las zonas donde se dan ataques en ramas y hojas, Australia o el sur de Italia, el hongo sobrevive y puede producir inóculo en éstas, por lo que el ciclo de patogénesis en estos lugares es diferente al que resulta de considerar exclusivamente los ataques al fruto (Martelli, 1960; Cacciola et al., 1996; Seergeva et al., 2008b).

La infección primaria ocurre durante la primavera pudiendo afectar a las inflorescencias aunque en condiciones de campo tienen escasa repercusión (Sergeeva et al., 2008a; Moral et al., 2009c). El patógeno también puede causar infecciones latentes (asintomáticas) en las aceitunas desde sus primeros estadios fenológicos (Moral et al., 2009c). Durante el otoño, cuando las aceitunas infectadas cambian de color, el patógeno se reactiva causando la típica podredumbre de aspecto jabonoso (Cacciola et al., 2012; Moral et al., 2009c). No se conocen los procesos que desencadenan la salida de latencia del patógeno, aunque las aceitunas 


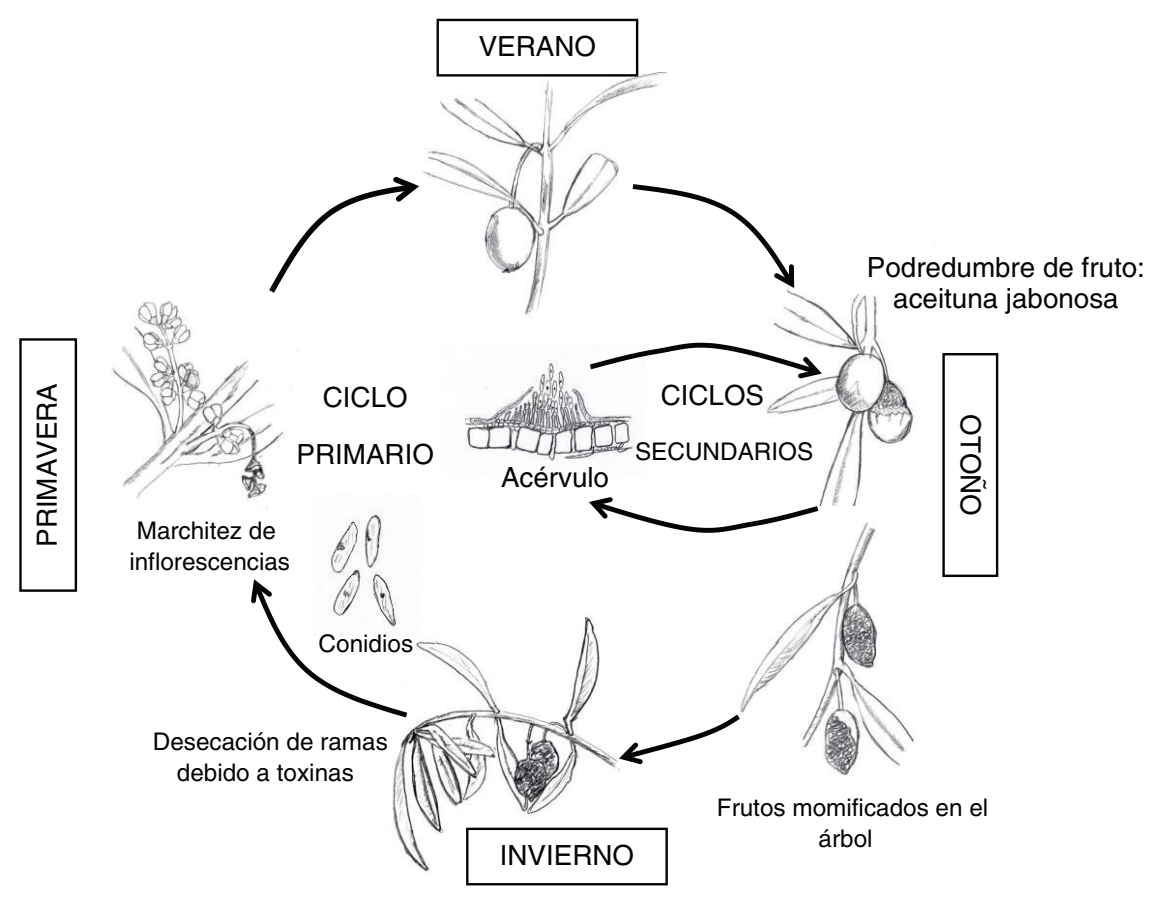

Figura 2. Ciclo de patogénesis de la Antracnosis del olivo causada por Colletotrichum spp.

disminuyen su resistencia con la madurez (Moral et al., 2008) debido a la dinámica de compuestos fenólicos del fruto. Las aceitunas momificadas que quedan en el árbol pueden producir inóculo a lo largo de todo el año cuando se hidratan. Así, tras las primeras lluvias de otoño se inicia la producción masiva de conidios en las momias. Una vez que el fruto está maduro, la Antracnosis muestra un periodo de incubación de 4-10 días en los cultivares susceptibles (Mateo-Sagasta, 1968; Graniti et al., 1993; Moral et al., 2008) por lo que se considera una enfermedad policíclica (Graniti et al., 1993; Trapero y Blanco, 2008; Cacciola et al., 2012; Figura 2). Aun así, en condiciones de campo suelen producirse pocos ciclos secundarios ya que cuando la temperatura media baja $\left(\leq 15^{\circ} \mathrm{C}\right)$ las infecciones se mantienen latentes (Moral y Trapero, 2012). Finalmente, el patógeno produce una toxina (Aspergillomarasmina B) en los frutos momificados que se moviliza causando el segundo síndrome: la desecación y muerte de ramas (Ballio et al., 1969; Bousquets et al., 1971; Bottalico, 1973; Moral et al., 2009c).

La temperatura óptima para la germinación de los conidios es $20-24{ }^{\circ} \mathrm{C}$ (Oliveira et al., 2005; Moral et al., 2011) aunque en algunos aislados Italianos se sitúa entre $25-30^{\circ} \mathrm{C}$ (Loprieno y Tenerini, 1960). La germinación, a su vez, es dependiente de la humedad relativa siendo necesaria agua libre o humedad próxima a saturación (>98\%) (Graniti et al., 1993; Moral et al., 2011). La infección de las aceitunas se produce a temperaturas comprendidas entre los $10 \mathrm{y}$
$30{ }^{\circ} \mathrm{C}$, mostrando un óptimo entre $17-20^{\circ} \mathrm{C}$, y se incrementa con el periodo de humectación (Graniti et al., 1993; Oliveira et al., 2005; Moral et al., 2012). La especie de Colletotrichum también influye en la infección y desarrollo de síntomas siendo $C$. simmondsii menos virulento que $C$. godetiae a temperaturas $\leq 20^{\circ} \mathrm{C}$ (Moral et al., 2012).

En Andalucía, la epidemia suele iniciarse durante la primera quincena de noviembre y se incrementa de forma exponencial hasta diciembre cuando la temperatura media baja $\left(\leq 15^{\circ} \mathrm{C}\right)$. Este hecho limita la salida de latencia del patógeno, de ahí que sea frecuente que la incidencia de aceitunas con infecciones latentes sea entre dos y tres veces superior al de aceitunas con lesiones visibles (Moral y Trapero, 2012; Figura 3). A su vez, la incidencia final está linealmente relacionada con tasa de maduración de las aceitunas, por lo que los síntomas se adelantan en olivos de cultivares susceptibles cuyas aceitunas maduran más rápido (Moral y Trapero, 2012; Figura 4). Este hecho está condicionado por la epidemia del año anterior, ya que si ésta causó la seca de ramos fructíferos, los olivos muestran menor carga de aceituna adelantando su maduración. Ello, junto a la presencia de mayor número de momias (inóculo), explicaría la existencia de epidemias concatenadas entre años (Mora et al., 2008). Finalmente, la severidad de la epidemia está relacionada con las precipitaciones acaecidas durante el otoño y no muestra relaciones con las ocurridas durante primavera (Moral y Trapero, 2010; Figura 5). 

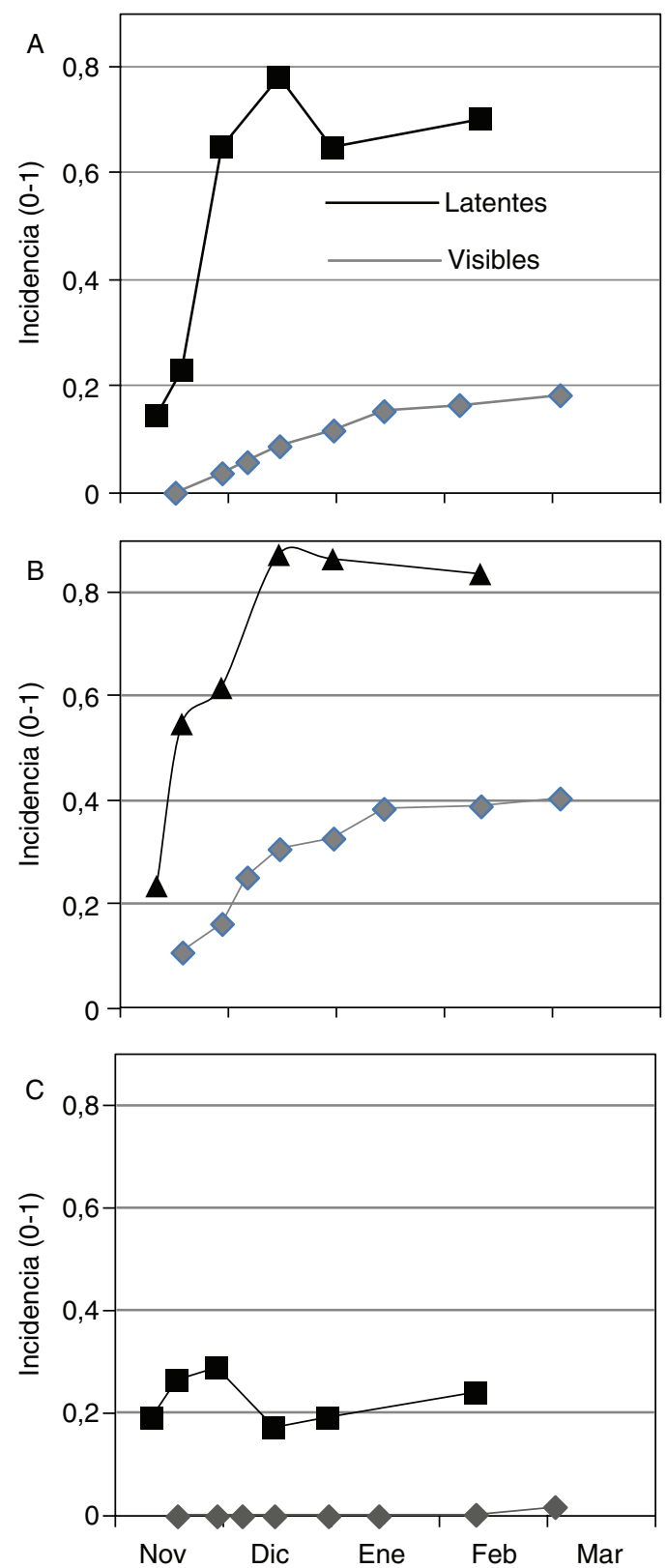

Figura 3. Evolución de la incidencia (0-1) de aceitunas con infecciones visibles y latentes de Colletotrichum spp. en el T. M de Cabra, Córdoba, durante la campaña 2005/06 en los cvs: (A) Hojiblanca (susceptible), (B) Picudo (susceptible) y (C) Picual (resistente).

En trabajos realizados en Andalucía con el cultivar moderadamente susceptible 'Arbequina', hemos podido observar el desarrollo epidémico de la enfermedad progresa más rápido en las plantaciones en seto (1904 árboles ha ${ }^{-1}$ ) que las plantaciones de alta densidad (204 a 816 árboles ha ${ }^{-1}$ ) (Moral et al., 2012). Este hecho se debe principalmente a las condiciones microclimáticas en el interior del seto (baja ventilación, elevada humedad relativa y tiempo de humectación) y al escasa distancia entre

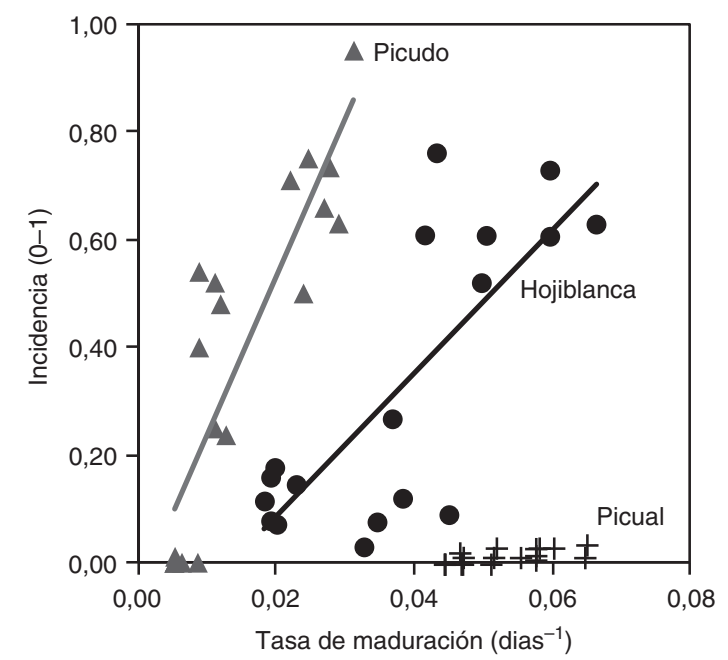

FIgURA 4. Relación lineal entre la tasa de maduración del fruto $\left(\right.$ días $\left.^{-1}\right)$ y la incidencia final $(0-1)$ de aceitunas afectadas por Colletotrichum spp. en los cvs. Hojiblanca (susceptible),

(B) Picudo (susceptible) y (C) Picual (resistente)
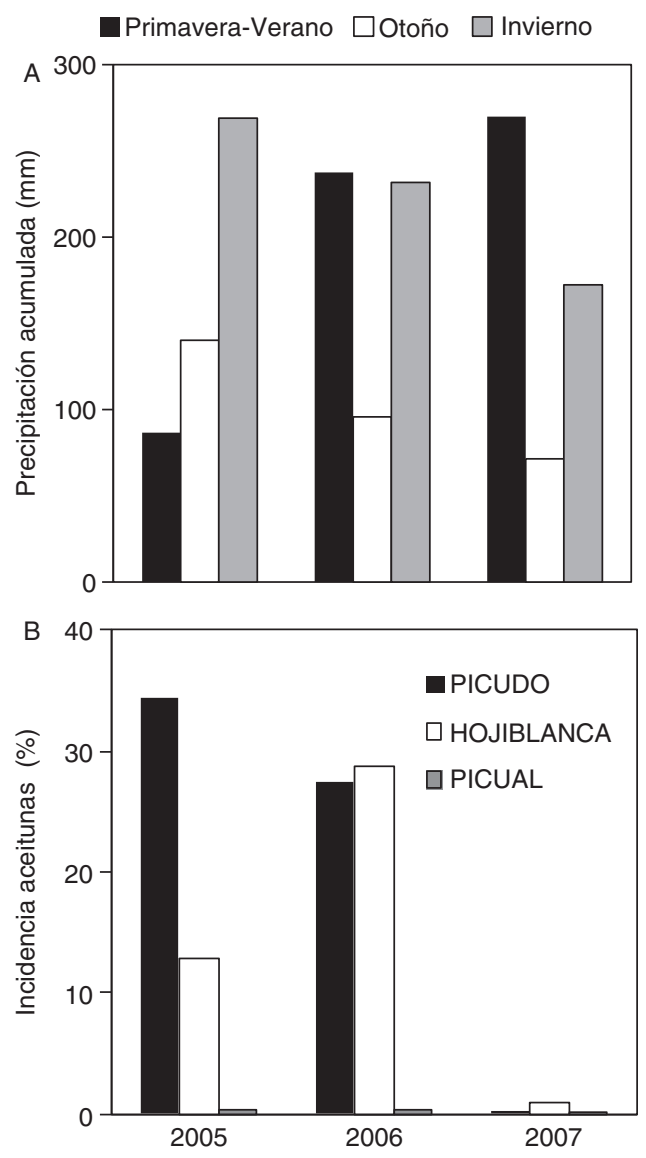

FIGURA 5. Relación entre precipitación e incidencia de aceitunas afectadas por Colletotrichum spp. durante 2005, 2006 y 2007 en el T. M de Cabra. (A) Precipitación acumulada ( $\mathrm{mm}$ ) en primavera-verano, otoño e invierno. (B) Aceitunas afectadas (\%) en los cvs. Hojiblanca (susceptible), Picual (resistente) y Picudo (susceptible). 
fuentes de inóculo (aceitunas momificadas), por lo que se recomienda incrementar los tratamientos cúpricos en este tipo de plantaciones.

Otros factores que influyen en el desarrollo epidémico de la enfermedad son: la presencia de microorganismos antagonistas (Segura, 2003), la presencia de heridas causadas por la Mosca del olivo (Mateo-Sagasta, 1968; Graniti et al., 1993; Moral et al., 2008), y los tratamientos fungicidas realizados (Roca et al., 2007). Además hemos podido observar que existe una relación negativa entre susceptibilidad a la Antracnosis y concentración de calcio en el fruto (Moral y Trapero, 2009b), lo que explicaría la severas epidemias que se desarrollan en suelos ácidos de Portugal o de las provincias de Sevilla y Huelva.

\section{CONTROL}

La resistencia es uno de los métodos de control más eficaces y respetuoso medioambientalmente, puede combinarse con otros métodos de control como el biológico o químico y ha permitido incrementar la producción agrícola amortiguando la fluctuación de la cosecha entre años. En el olivo, desafortunadamente, la resistencia a plagas o enfermedades no se ha considerado como una medida de control debido en gran parte a la falta de una información rigurosa sobre la resistencia/susceptibilidad de los cultivares. En el caso de la Antracnosis del olivo, la resistencia y susceptibilidad genética son los extremos de una variable continua resultado de la interacción entre los mecanismos utilizados por el hongo para producir infección y enfermedad y los mecanismos, pasivos o activos, de resistencia de la planta (Moral y Trapero, 2009b).

En condiciones controladas, la resistencia del olivo a Colletotrichum spp. se expresa como: retraso en la colonización de los tejidos de la aceituna (Gomes et al., 2009), incremento del periodo de latencia, disminución de la tasa máxima de desarrollo de síntomas (Moral et al., 2008) y reducción de la cantidad de inóculo que produce el patógeno (Moral y Trapero, 2012). En campo, se observa una disminución de la tasa máxima de desarrollo de síntomas y un incremento del periodo de latencia, siendo frecuente que las infecciones queden asintomáticas durante periodos prolongados en los cultivares resistentes (Moral y Trapero, 2012; Figura 3).

Actualmente podemos encontrar desde cultivares de olivo altamente susceptibles a cultivares con resistencia completa (Tabla 2) aunque esta información presenta limitaciones, ya que en la mayoría de los casos está basada en observaciones de campo siendo frecuentes las contradicciones debido a errores de identificación de los cultivares y a la confusión entre síntomas causados por distintos patógenos (MateoSagasta, 1968; Moral et al., 2005). Un factor crítico que condiciona la resistencia de los cultivares es el estado de madurez del fruto, ya que cuando la aceituna está sobremadura todos los cultivares resultan susceptibles y apenas existen diferencias entre ellos. Otros factores que pueden influir en la resistencia son la interacción especie de Colletotrichum-cultivar o a la existencia de grupos de virulencia o razas del patógeno (Moral y Trapero, 2009b; Xaviér et al., 2010).

La evaluación de los 15 primeros cultivares obtenidos en el programa de mejora UCO-IFAPA de Córdoba también ha permitido encontrar una amplia respuesta a la Antracnosis, obteniéndose dos cultivares resistentes ('UC 07-60' y 'UC 10-30'), aunque ninguno mejora las características de resistencia del parental 'Frantoio' (Moral et al., 2006).

Por último, cabe comentar que tanto en las prospecciones de campo como en las inoculaciones artificiales hemos podido observar que la mayoría de los genotipos de acebuche muestran una elevada resistencia a la Antracnosis. Esta selección natural hacia resistencia puede deberse al hecho de que el patógeno afecta a la viabilidad de la semillas (Xaviér et al., 2012) o la capacidad de dispersión de las aceitunas sintomáticas que podrían ser rechazadas por las aves frugívoras.

Las prácticas culturales que favorezcan la ventilación de los olivos, como la poda de las ramas internas y evitar los marcos de plantación excesivamente estrechos, muestran elevada eficacia en la Antracnosis del olivo. Además, es aconsejable la eliminación de las ramas muertas y de las aceitunas momificadas de la copa de los árboles. Cabe destacar, que la estrategia de control más efectiva es adelantar la recolección, debido a que la susceptibilidad de la aceituna aumenta con su estado de madurez, acortándose el tiempo necesario para que se desarrollen ciclos secundarios (Andrés, 1991; Graniti et al., 1993; Moral et al., 2008; Trapero et al., 2009; Cacciola et al., 2012). Este hecho, ha llevado a algunos autores a recomendar la utilización de cultivares de maduración tardía (Bompeix et al., 1988).

En cultivares susceptibles a la Antracnosis, cuando no se recogen en verde, el control efectivo de la enfermedad requiere el empleo de fungicidas. A pesar de que se redujo un $43 \%$ el consumo de fungicidas cúpricos a nivel europeo durante 1993-2003 en favor de otros compuestos como los carbamatos, quinolinas o estrobilurinas (Eurostat, 2007), la aplicación de fungicidas cúpricos sigue siendo la medida de control más utilizada para la enfermedades del olivo y en particular, para la Antracnosis (Martelli y Piglionica, 1961; Graniti y Laviola, 1981; Graniti et al., 1993; Pérez, 2011), y suponen un gasto anual de unos 200 millones de euros al sector oleícola español (Trapero et al., 2009). Esta prevalencia de los productos cúpricos se debe a que el uso de fungicidas orgánicos en postfloración está muy limitado ya que existe la posibilidad de que se absorban en el aceite del fruto al ser en su mayoría liposolubles (Moral y Trapero, 2009b). 
La Antracnosis del olivo y su efecto en la calidad del aceite $\bullet 9$

TABLA 2. Resistencia de cultivares de olivo a la Antracnosis causada por Colletotrichum spp

\begin{tabular}{|c|c|c|c|}
\hline Cultivar & Reacción $^{\mathrm{a}}$ & Cultivar & Reacción ${ }^{a}$ \\
\hline Abou-salt & $\mathrm{R}^{3(\mathrm{~b})}$ & Manzanilla del centro & $\mathrm{S}^{16}$ \\
\hline Alameño de Marchena & $\mathrm{R}^{16}$ & Manzanilla del piquito & $S^{16}$ \\
\hline Alfafara & $S^{16}$ & Megaritiki & $\mathrm{R}^{16}$ \\
\hline Arauco & $\mathrm{R}^{3}$ & Manzanilla del centro & $S^{16}$ \\
\hline Arbequina & $\mathrm{S}^{2,4} \mathrm{M}^{7-14-16-19}$ & Meski & $S^{16}$ \\
\hline Arbosana & $\mathrm{R}^{16-19}$ & Mele & $\mathrm{S}^{4}$ \\
\hline Ascolana tenera & $\mathrm{S}^{4}$ & Mission & $\mathrm{S}^{4-19} \mathrm{M}^{4}$ \\
\hline Azara & $\mathrm{S}^{20}$ & Mixan & $\mathrm{M}^{4}$ \\
\hline Azeiteira & $\mathrm{R}^{5}$ & Moraiolo & $\mathrm{S}^{4}$ \\
\hline Azeitoneira & $\mathrm{M}^{4}$ & Morisca & $S^{4-15-16}$ \\
\hline Barnea & $S^{4-19-20}$ & Morona & $\mathrm{S}^{19}$ \\
\hline Barouni du nord & $\mathrm{S}^{4}$ & Morrudo & $S^{4-7}$ \\
\hline Bical de Castelo Branco & $\mathrm{M}^{4}$ & Morrut & $\mathrm{S}^{4-7} \mathrm{M}^{16-19}$ \\
\hline Bico de corno & $S^{2}$ & Negrinha & $M^{4} R^{5}$ \\
\hline Blanqueta & $\mathrm{S}^{16} \mathrm{M}^{4-5} \mathrm{R}^{15-19}$ & Nera di Oliena & $\mathrm{M}^{4}$ \\
\hline Blanqueta de Elvas & $\mathrm{R}^{5}$ & Nabali & $\mathrm{R}^{16}$ \\
\hline Branquita & $\mathrm{R}^{4}$ & Nevadillo blanco de Lucena & $S^{14}$ \\
\hline Callosina & $\mathrm{S}^{16} \mathrm{R}^{14}$ & Negral & $\mathrm{M}^{14}$ \\
\hline Canetera & $\mathrm{R}^{7}$ & Nocettara Etnea & $S^{8}$ \\
\hline Carolea & $S^{19}$ & Ocal & $\mathrm{S}^{16-19}$ \\
\hline Carrasquenha & $\mathrm{R}^{4-5}$ & Ogliorola di Lecce & $M^{13}$ \\
\hline Cassanese & $\mathbf{M}^{19}$ & Orebetana & $\mathrm{R}^{4}$ \\
\hline Cellina di Nardo & $\mathrm{S}^{12} \mathrm{M}^{7}$ & Ottobrarica & $\mathrm{S}^{12-19}$ \\
\hline Changlot real & $\mathrm{S}^{14-16}$ & Ottobratica rotondella & $\mathrm{S}^{4}$ \\
\hline Cipressino & $S^{16}$ & Pajarero & $\mathrm{M}^{19} \mathrm{R}^{16}$ \\
\hline Cobrançosa & $S^{4-5-16}$ & Patronet & $\mathbf{M}^{7}$ \\
\hline Conserva de Elvas & $S^{4-5}$ & Pendolino & $\mathrm{S}^{11}$ \\
\hline Coratina & $\mathrm{M}^{4}$ & Pequeña de Casa Ibañez & $\mathrm{R}^{16}$ \\
\hline Cordovil de Castelo Branco & $\mathrm{S}^{5} \mathrm{M}^{4}$ & Perillo de Jaén & $\mathrm{R}^{16}$ \\
\hline Cornezuelo de Jaén & $\mathrm{R}^{14}$ & Picholine & $\mathrm{S}^{3} \mathrm{R}^{16}$ \\
\hline Cornicabra & $S^{14-16}$ & Picholine marrocaine & $\mathrm{R}^{16}$ \\
\hline Cornicabra de Mérida & $S^{16}$ & Picual & $\mathrm{R}^{5-14-16-19}$ \\
\hline Cornicabra parda & $\mathrm{R}^{14}$ & Picual de Estepa & $S^{16}$ \\
\hline Corniola & $\mathrm{S}^{11-19}$ & Picual de hoja clara & $S^{16}$ \\
\hline Dolce Agogia & $\mathrm{R}^{16}$ & Picudo & $\mathrm{S}^{3-14-16}$ \\
\hline Empeltre & $\mathbf{M}^{3-14} \mathbf{R}^{7-16-19}$ & Picudo de Montoro & $S^{16}$ \\
\hline Farga & $\mathrm{S}^{14-16} \mathrm{M}^{7}$ & Queen & $S^{10}$ \\
\hline Frantoio & $\mathrm{S}^{2-11} \mathrm{M}^{4} \mathrm{R}^{5-15-16-19}$ & Racimal de Jaén & $S^{16}$ \\
\hline FS-17 & $\mathrm{R}^{4}$ & Razzola & $\mathrm{R}^{19}$ \\
\hline Galega grada de Serpa & $S^{4}$ & Rasti & $\mathrm{S}^{4}$ \\
\hline Galega vulgar & $\mathrm{S}^{2-5-16}$ & Redondal & $S^{4-5}$ \\
\hline Gerboui du nord & $\mathrm{R}^{4}$ & Redondil & $\mathrm{S}^{4}$ \\
\hline Gordal sevillana & $\mathrm{S}^{14-16}$ & Picual de hoja clara & $S^{16}$ \\
\hline Hojiblanca & $\mathrm{S}^{14-16}$ & Redondilla de Logroño & $S^{14}$ \\
\hline Itrana & $S^{4-19}$ & San Mauro & $\mathrm{S}^{12}$ \\
\hline Kalamata & $\mathrm{S}^{20}$ & Sant'Agostino & $\mathrm{S}^{4}$ \\
\hline Kalinjot & $\mathrm{M}^{4}$ & Santomauro & $\mathrm{R}^{19}$ \\
\hline
\end{tabular}




\begin{tabular}{|c|c|c|c|}
\hline Kokërr Madh Berati & $S^{4}$ & Sevillenca & $S^{7-16}$ \\
\hline Kokërr Madh Elbasani & $\mathrm{M}^{4}$ & Sikitita (=Chiquitita) & $\mathrm{S}^{19}$ \\
\hline Koroneiki & $\mathrm{R}^{16-19}$ & Sinopolese & $S^{6-12-19}$ \\
\hline Leccino & $\mathrm{S}^{11-16} \mathrm{M}^{19} \mathrm{R}^{19}$ & Tempranilla & $\mathrm{R}^{14}$ \\
\hline Lechín de Granada & $S^{16}$ & Tonda di Strongoli & $\mathrm{S}^{4}$ \\
\hline Lechín de Sevilla & $\mathrm{S}^{14-16}$ & Tonda Iblea & $S^{4-8-9}$ \\
\hline Leucocarpa & $\mathrm{M}^{4}$ & Tondina & $\mathrm{S}^{19}$ \\
\hline Limli & $\mathrm{M}^{4}$ & Verdeal Alentejana & $M^{4} R^{5}$ \\
\hline Llumet & $\mathrm{R}^{7}$ & Verdial de Badajoz & $S^{3}$ \\
\hline Maçanilha algarvia & $S^{4-5}$ & Verdial de Huevar & $S^{16} R^{14}$ \\
\hline Manzanilla cacereña & $\mathrm{M}^{16}$ & Verdial de Vélez-Málaga & $\mathrm{S}^{14} \mathrm{M}^{16}$ \\
\hline Manzanilla de agua & $S^{16}$ & Vernina (=Ciparsiota) & $\mathrm{S}^{12}$ \\
\hline Manzanilla de Carmona & $\mathrm{M}^{3}$ & Villalonga & $\mathrm{S}^{16} \mathrm{M}^{19}$ \\
\hline Manzanilla de Guadix & $S^{16}$ & Zarzaleña & $\mathrm{R}^{14}$ \\
\hline Manzanilla de Hellín & $\mathrm{R}^{16}$ & Zarzariega & $S^{14}$ \\
\hline Manzanilla de Jaén & $\mathrm{S}^{16} \mathrm{R}^{14}$ & Zutica & $M^{4} R^{3}$ \\
\hline Manzanilla de Sevilla & $\mathrm{S}^{2-3-7-14-16-20}$ & UC $13 \mathrm{~A} 6$ & $\mathrm{~S}^{20}$ \\
\hline Manzanilla de Tortosa & $S^{16}$ & - & - \\
\hline \multicolumn{4}{|c|}{ 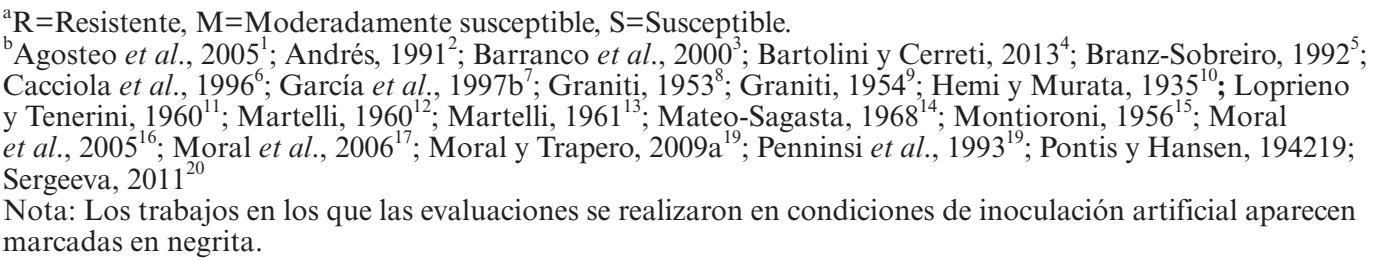 } \\
\hline
\end{tabular}

Los compuestos cúpricos además poseen numerosas características que han motivado su amplia utilización como: elevada persistencia, amplio espectro de acción contra hongos y bacterias, capacidad de interferir con las toxinas del patógeno y precio bajo, aunque en los últimos años se ha incrementado su precio sustancialmente (Pennissi et al., 1993, Roca et al., 2007). Asimismo, y aunque se han utilizado en el olivar prácticamente desde su descubrimiento por Millardet (1885), no se han detectado poblaciones de hongos tolerantes, al tratarse de fungicidas " $m u l$ tisitio" que afectan a la membrana celular de las esporas sustituyendo otros iones $\left(\mathrm{H}^{+}, \mathrm{K}^{+}, \mathrm{Ca}^{2+} \mathrm{y}\right.$ $\mathrm{Mg}^{2}$ ) esenciales y afectando a las esterasas de membrana. En el interior de la espora, los iones $\mathrm{Cu}^{2+}$ se fijan sobre diversos grupos químicos (imidazoles, carboxilos, fosfatos, sulfhidrilos, aminas o hidroxilos) presentes en numerosas proteínas y afectan a la cadena de respiración mitocondrial (Montag et al., 2006; Borkow y Gabbay, 2009).

En un trabajo realizado durante 4 años en Andalucía, se identificó el inicio del otoño como el momento óptimo de control de la enfermedad (Pérez, 2011) coincidiendo con las recomendaciones realizadas en Italia (Graniti et al., 1993; Penninsi et al., 1993; Prota, 1995). Debido a la enorme capacidad de dispersión y multiplicación del hongo y al carácter protector de los fungicidas cúpricos, los tratamientos otoñales deben realizarse antes de que se observe el primer fruto afectado en campo, repitiéndose en años lluviosos o con cultivares tardíos debido al lavado del cobre (Moral et al., 2009b). Por el contrario, los tratamientos otoñales no son necesarios en las zonas donde predominen cultivares resistentes a la Antracnosis, como es el caso de la provincia de Jaén donde domina el cv. Picual. Hasta el momento, los tratamientos primaverales han mostrado una eficacia limitada (Martelli y Piglionica 1961; Pérez, 2011) debido a la mayor importancia de las infecciones otoñales. Por ello, estos tratamientos sólo serían eficaces los años con primavera lluviosa, elevado inóculo en campo y otoño seco.

En general, los años con elevado inóculo en campo (años precedidos de epidemias de Antracnosis), condiciones climáticas favorables (otoño húmedo y cálido) para el desarrollo de le enfermedad y en los que las aceitunas adelante su maduración (años de descarga), si los cultivares son susceptible al patógeno, es recomendable mantener protegido el fruto durante todo el otoño y adelantar la recolección. En el caso de que el cultivar sea de doble aptitud (mesa y aceite) podemos optar por el verdeo.

Los compuestos cúpricos que hemos evaluado (hidróxido de cobre, oxicloruro de cobre y sulfato cuprocálcico) han mostrado un eficacia alta contra el patógeno a excepción del sulfato tribásico de 
cobre. A nivel experimental, también hemos observado una elevada eficacia del tebuconazol, aunque su uso está limitado a prefloración (Pérez, 2011). En Italia, Pennisi et al. (1993) observaron que el sulfato cuprocálcico permite un control mejor de la enfermedad que el bitertanol, hexaconazol, miclobutanil metiram y ziram. Resultados similares se obtuvieron en la India al comparar los compuestos cúpricos con distintos compuestos orgánicos (Sharma y Kaul, 1990). En el caso de las estrobilurinas, familia de fungicidas de más reciente incorporación al olivar, han sido escasamente investigadas contra la Antracnosis, aunque existen resultados que demuestran la eficacia de azoxistrobin en Italia (Agosteo et al., 2007) y de trifloxistrobin en España (Pérez, 2011). Cabe destacar que se han observado buena eficacia de la mezclas de compuestos cúpricos y orgánicos, como en el caso de oxicloruro de cobre y propineb o trifloxistrobin, o hidróxido cúprico y folpet (Pérez, 2011). Actualmente, el estudio de mezclas de ambos tipos de fungicidas, o la utilización de nuevos compuestos con baja concentración de cobre, son de especial interés ya que se espera una reducción importante de la cantidad cobre permitida que actualmente es de $6 \mathrm{~kg} / \mathrm{ha}$ al año en la Unión Europea (EEC, 2007; Trapero et al., 2009; Cacciola et al., 2012).

Los compuestos cálcicos se están utilizando para control de la Antracnosis del manzano debido a que inhiben la actividad de enzimas excretadas por los patógenos y refuerzan la estructura de la pared celular de los tejidos del fruto (Rahman y Punja, 2007). En el caso del olivo, aún no hemos observado un efecto destacable de los compuestos de calcio (carbonato, cloruro, hidróxido, propinato y silicato) en el control de la enfermedad (Pérez, 2011). Aunque alguno de estos compuestos causan una inhibición de la formación de los apresorios, estructura fundamental para la infección de los conidios, próxima al 80\% (Agalliu, 2009). Además, en condiciones naturales, se ha observado que el contenido de calcio en la aceituna esta positivamente relacionado con la resistencia que muestra al patógeno (Moral et al., 2009b).

La lucha biológica no han sido empleada de forma comercial contra la Antracnosis del olivo, aunque en inoculaciones artificiales de aceitunas, un aislado fúngico de Aureobasidium pullulans y dos bacterianos, Curtobacterium flaccumfaciens y Paenibacillus polymyxa, mostraron una capacidad de inhibición superior al 50\% (Segura, 2003).

\section{INFLUENCIA SOBRE LA CALIDAD DEL ACEITE}

La obtención de un aceite de calidad es una premisa fundamental para cualquier olivicultor. Para ello es necesario tener en cuenta los diferentes factores que pueden afectar a la calidad del producto final en cualquiera de sus etapas de producción. Los factores climatológicos, edafológicos, agronómicos y, especialmente, la recolección y la sanidad de la aceituna influyen notablemente en la calidad del aceite de oliva.

Los parámetros físico-químicos (acidez libre, índice de peróxidos, absorbancia ultravioleta K270 y K232, y ésteres alquílicos), y sensoriales que permiten diferenciar las categorías comerciales del aceite de oliva virgen están recogidos en los reglamentos EEC 1991 y IOOC 2012 (Tabla 3). A su vez, los aceites de oliva de mayor calidad (vírgenes extras) se diferencian según la cantidad e intensidad de matices sensoriales tanto para el olfato como para el gusto.

En general, los aceites procedentes de aceitunas caídas muestran elevada acidez y deterioro de la calidad sensorial (Uceda et al., 2008). Si las aceitunas caídas suponen un $5-10 \%$ del total de frutos, se produce un descenso notable de la calidad del aceite (Famiani et al., 2002). El estado de madurez de la aceituna también tiene una marcada influencia en la calidad del aceite (Alba et al., 2008). En general, el contenido en compuesto fenólicos se incrementa durante las primeras fases de maduración de la aceituna y disminuye posteriormente según una curva parabólica (Uceda et al., 2008). Durante el la maduración, además, se suele producir un incremento del ácido linoleico y, en ocasiones, del oleico, mientras que los ácidos palmítico y esteárico tienden a disminuir causando un descenso de la relación monoinsaturados/poliinsaturados (Uceda et al., 2008; Inglese et al., 2011). Para establecer el momento óptimo de recolección se aconseja la realización de análisis periódicos de las aceitunas (Alba, 2008), aunque suele ser próximo al momento en el que la pigmentación está limitada a la epidermis (Inglese et al., 2011). Por lo tanto, los sistemas de recolección que permitan una recolección de aceitunas aéreas en el momento óptimo de madurez de forma rápida, para evitar la sobremaduración y caída, y que no dañen su epidermis son los más aconsejados. Entre estos sistemas, podemos destacar los vibradores, tanto de tronco como manuales, y la vendimiadoras utilizadas para los setos del olivar de alta densidad. Recientemente, Guerfel et al $(2010 ; 2012)$ demostraron que este factor agronómico, el marco de plantación, también puede afectar a las características químicas (ácido oleico, hexanol, clorofila, carotenoides y fenoles totales) aunque estos estudios se realizaron en condiciones de secano en Túnez y con densidades comprendidas entre $51 \mathrm{y}$ 156 árboles ha ${ }^{-1}$.

Una vez recogida la aceituna, los procesos de transporte, almacenamiento de las aceitunas, batido, centrifugación y almacenamiento del aceite también influyen marcadamente en la calidad del aceite (Alba, 2008). 
TABLA 3. Características físico-químicas y sensoriales de las diferentes categorías de aceite de oliva

\begin{tabular}{|c|c|c|c|c|}
\hline Clasificación & Acidez Libre (\%) & P.V. (meq $\left.\mathrm{O}_{2} \mathrm{~kg}^{-1}\right)$ & UV 270 nm & $\begin{array}{c}\text { C. Organolépticas } \\
\text { Intensidad Defecto; Frutado }\end{array}$ \\
\hline Virgen Extra & $\leq 0,8$ & $\leq 20$ & $\leq 0,22$ & $\begin{array}{l}\text { Defecto }=0 \\
\text { Frutado }>0\end{array}$ \\
\hline Virgen & $\leq 2,0$ & $\leq 20$ & $\leq 0,25$ & $\begin{array}{l}0<\text { Defecto } \leq 3,5 \\
\text { Frutado }>0\end{array}$ \\
\hline Virgen Corriente ${ }^{a}$ & $\leq 3,3$ & $\leq 20$ & $\leq 0,30$ & $\begin{array}{l}3,5<\text { Defecto } \leq 6,0 \\
0<\text { Defecto } \leq 3,5 \text { y } F=0\end{array}$ \\
\hline Lampante & $>2,0>3,3^{b}$ & - & - & $\begin{array}{l}\text { Defecto }>3,5 \\
0<\text { Defecto } \leq 3,5 \text { y } F=0 \\
\text { Defecto }>6,0^{6}\end{array}$ \\
\hline
\end{tabular}

${ }^{\mathrm{a}}$ Sólo existe esta categoría en el Reglamento COI T. 15/NC N³/Rev. 7

${ }^{\mathrm{b}}$ Valor para dicha categoría en el Reglamento COI T. 15/NC N³/Rev. 7.

La obtención de aceite de oliva calidad requiere la utilización de aceitunas sanas, es decir que no estén afectadas por insectos u organismos patógenos que les causen alteraciones (Runcio et al., 2008). Entre los insectos, destaca la Mosca del olivo que puede causar tanto pérdida de cosecha como de calidad del aceite, debido a la caída de aceitunas y a la galerías de las larvas en la pulpa de los frutos (Andrés, 1991). Una elevada infestación de Mosca está asociada con incrementos de la acidez libre, de la absorbancia en el ultravioleta y del índice de peróxidos (Tamendjari et al. 2009; Mraicha et al., 2010), así como una disminución de la estabilidad y contenido de compuestos fenólicos del aceite (Gómez-Caravaca et al. 2008; Tamendjari et al. 2009; Mraicha et al., 2010). En la mayor parte de los casos, los agentes biológicos que en última instancia deterioran la calidad del aceite son hongos filamentosos o levaduras que colonizan las galerías de las larvas de la mosca (Andrés, 1991).

Entre los organismos patógenos de las aceitunas destacan diversos hongos y principalmente las especies del género Colletotrichum (García et al., 1997a; García, 1998). Estudios recientes han mostrado que aceitunas bien recogidas y procesadas producen

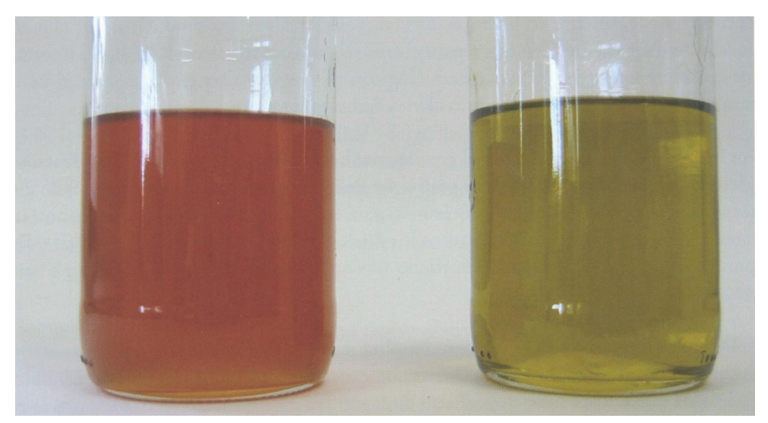

FIGURA 6. Aceite de oliva "colorado" (izq.) procedente de aceitunas afectadas por Colletotrichum spp. junto a aceite virgen extra (dcha.) procedente de aceitunas sanas (Foto de: Miguel Pastor). aceites de inferior calidad a las procesadas de manera irregular, siendo sólo la diferencia entre ellas la incidencia en las primeras de frutos afectados por Antracnosis. Las especies de Colletotrichum causan la podredumbre y caída prematura de las aceitunas, lo que origina en el aceite un color rojizo ("aceites colorados") característico (Figura 6), aumento de la acidez y pésima calidad organoléptica, apareciendo los defectos de tierra y moho-húmedo-terroso (Mincione et al., 2004; Carvalho et al., 2008).

En general, a medida que aumenta la incidencia de frutos afectados por Antracnosis, se produce simultáneamente una caída de la calidad de los aceites extraídos. Los aceites procedentes de aceitunas afectadas muestran aumento en el índice de peróxidos y, sobre todo, en la acidez libre (Iannotta et al., 1999); y una disminución de la estabilidad oxidativa, del contenido de polifenoles y $\alpha$-tocoferol. De manera, que aceites procedentes de aceitunas con una incidencia del 15-20\% dejan de ser clasificados como aceites de oliva virgen extra y con una incidencia mayor del $40-45 \%$ no pueden ser clasificados como aceites vírgenes al mostrar un porcentaje de acidez $>0,8 \%$ y $2 \%$, respectivamente (Mincione et al., 2004; Carvalho et al., 2008).

Otro parámetro de caracterización del aceite afectado por la Antracnosis es la composición esterólica que puede impedir el cumplimiento de las normas de comercio internacional de los aceites de oliva. En cambio, la composición de ácidos grasos se mantiene más o menos estable (Iannotta et al., 1999; Mincione et al., 2004) (Tabla 4).

Recientemente, Runcio et al. (2008) han demostrado que los aceites procedentes de aceitunas afectadas por el patógeno muestran un incremento significativo en el contenido de aldehídos, principalmente heptanal, octanal y nonanal que atribuyen a las reacciones de descomposición de hidróxidos peróxidos que se forman durante la auto-oxidación del ácido oleico del aceite (Vichi et al., 2003). 
TABla 4. Efecto de la incidencia (\%) de aceitunas cv. Sinopolese afectadas por Colletotrichum en la calidad del aceite.

\begin{tabular}{|c|c|c|c|c|c|c|c|c|}
\hline Incidencia (\%) & $\begin{array}{c}\text { Acidez } \\
\text { Libre (\%) } \\
\end{array}$ & $\begin{array}{l}\text { Índice Peróxidos } \\
\left(\text { meq } \mathrm{O}_{2} \mathrm{Kg}^{-1}\right)\end{array}$ & K232 & K270 & $\begin{array}{c}\text { Estabilidad } \\
\text { Oxidativa (h) } \\
\end{array}$ & $\begin{array}{c}\text { Fenoles Totales } \\
\left(\mathrm{mg} \mathrm{Kg}^{-1}\right)\end{array}$ & $\begin{array}{c}\beta \text {-Sitosterol } \\
(\%)\end{array}$ & $\begin{array}{c}\text { Esteroles Totales } \\
\left(\mathrm{mg} \mathrm{kg}^{-1}\right)\end{array}$ \\
\hline 0 & 0,45 & 4,16 & 1,40 & 0,13 & 11,00 & 299,7 & 93,9 & 3026 \\
\hline 20 & 2,58 & 16,24 & 1,50 & 0,15 & 4,50 & 208,7 & 93.4 & 3443 \\
\hline 40 & 6,43 & 33,14 & 1,75 & 0,19 & 2,00 & 139,7 & 91.6 & 4197 \\
\hline 60 & 6,00 & 32,80 & 1,77 & 0,19 & 2,50 & 148,8 & 91.8 & 4400 \\
\hline 100 & 8,38 & 42,73 & 2,06 & 0,25 & 1,50 & 133,4 & 90.8 & 4855 \\
\hline
\end{tabular}

Fuente: Iannotta et al., 1999.

TABla 5. Contenido en ésteres alquílicos de los ácidos grasos de aceites obtenidos de aceitunas con diferentes grados de deterioro

\begin{tabular}{|c|c|c|c|c|c|}
\hline Muestra & Apilamiento (días) & FAME $\left(\mathrm{mg} \mathrm{kg}^{-1}\right)$ & FAEE $\left(\mathrm{mg} \mathrm{kg}^{-1}\right)$ & FAPE $\left(\mathrm{mg} \mathrm{kg}^{-1}\right)$ & FABE $\left(\mathrm{mg} \mathrm{kg}^{-1}\right)$ \\
\hline Inicial & 0 & 24 & 18 & n.d. & n.d. \\
\hline 1 & 14 & 24323 & 177703 & 434 & 905 \\
\hline 2 & 28 & 24963 & 88925 & 380 & 693 \\
\hline 3 & 42 & 30014 & 167783 & 735 & 1052 \\
\hline 4 & 50 & 38923 & 278260 & 1027 & 1543 \\
\hline
\end{tabular}

Fuente: Pérez-Camino et al., 2002.

Asimismo, la infección por Colletotrichum también afecta a los alcoholes alifáticos y terpénicos y los contenidos de ceras (Mincione et al., 2004).

Por último, el contenido de ésteres alquílicos de los ácidos grasos en los aceites obtenidos de aceitunas que han sufrido un proceso de degradación por apilamiento en condiciones húmedas durante un largo período aumenta considerablemente (Tabla 5). Por lo que cabe esperar que la incidencia de Colletotrichum spp., que produce un aumento de la acidez libre y del contenido de alcoholes alifáticos, origine un aumento por esterificación de los ácidos grasos y los alcoholes, de manera que posiblemente no sólo haya ésteres metílicos y etílicos sino que se produzcan otros como propílicos y butílicos (Gómez-Coca et al., 2012).

\section{PERSPECTIVAS FUTURAS}

Aunque durante los últimos años se han realizado avances importantes en el conocimiento de la epidemiología y control de la Antracnosis del olivo, todavía quedan numerosas investigaciones por desarrollar para conseguir un control más eficaz y respetuoso medioambientalmente. Por ello, actualmente estamos trabajando en el desarrollo de un sistema de toma de decisiones con un entorno informático amigable para los olivicultores. Con este sistema se espera identificar los momentos óptimos de control de la enfermedad en función de las condiciones ambientales y de la resistencia del cultivar lo que derivaría en un ahorro sustancial de fungicidas.
En el caso de la influencia del patógeno en la calidad del aceite de oliva, y a pesar de sus nefastas consecuencias, las carencias de conocimiento son aún mayores. Creemos que son necesarios estudios que aborden la influencia de la incidencia y severidad de la infección sobre la calidad del aceite incluyendo, además de los parámetros tradicionales, como la acidez y el índice de peróxidos, otros como el contenido de ésteres. Igualmente interesante sería estudiar el efecto del patógeno sobre la calidad de los aceites elaborados con aceitunas afectadas por el patógeno pero que aún no muestran síntomas visibles, es decir durante el proceso de infección latente. Por último, creemos esencial abordar el efecto varietal y etiológico en la calidad de los aceites procedentes de frutos afectados y la dinámica de degeneración de los aceites colorados. Afrontar estos trabajos requiere la colaboración necesaria de químicos del aceite y fitopatólogos.

\section{AGRADECIMIENTOS}

Las investigaciones realizadas han sido financiadas por los proyectos AGL2000-1725 y AGL2004 7495 del Ministerio de Educación y Ciencia y P08AGR-03635 de la Junta de Andalucía. Juan Moral es contratado postdoctoral del programa Juan de la Cierva. Carlos Xaviér ha sido becario de la AECI durante tres años. Expresamos nuestro agradecimiento a todos los investigadores y agricultores que de un modo $u$ otro han hecho posible este trabajo. Igualmente agradecemos a los revisores anónimos y a Marta Aguirre sus sugerencias. 


\section{REFERENCIAS}

Agalliu G. 2009. Evaluación de Fungicidas para el control de la antracnosis del olivo causada por Colletotrichum acutatum. Trabajo profesional fin de Máster, ETSIAM, Córdoba.

Agosteo GE, Li Destri Nocosia, MG, Magnano di San Lio G, Frisullo S, Cacciola SO. 2000. Characterization of the causal agent of olive Anthracnose in southern Italy. IV Symposium on Olive Growing, Bari (Italia).

Agosteo GE, Macrì C, Cacciola SO, Magnano di San Lio G. 2006. Olive fruit fly (Bactrocera oleae) is a vector of Colletotrichum sp. causing olive anthracnose. XIII Congresso SIPV. Foggia, (Italia).

Agosteo GE, Macri C, Taccone P. 2005. Susceptibility of olive cv. Itrana to Anthracnose. J. Plant Pathol. 87, 287.

Agosteo GE, Scolaro L, Previtera G. 2007. Non-conventional chemical control of olive anthracnose. Integrated protection of Olive Crops. IOBC/WPRS Bull. 30, 245-248.

Ainsworth GC. 1973. Introduction and keys to higher taxa. In: Ainsworth GC, Sparrow FK, and Sussman AS (Eds). The fungi: an advanced treataise. Academic Press, Nueva York, $1-7$.

Alba J. 2008. Elaboración de aceite de oliva virgen. In Barranco D, Fernández-Escobar R, Rallo L (Eds) El cultivo de olivo. Coedición Junta de Andalucía/Mundi-Prensa, Madrid, 657-697.

Almeida MJV. 1899. La gaffa des olives en Portugal. Bull. Soc. Mycol. Franc. 15, 90-94.

Andrés F. 1991. Enfermedades y plagas del olivo. $2^{\mathrm{a}} \mathrm{Ed}$. Riquelme y Vargas Ediciones, S.L., Jaén.

Arx von JA. 1970. A revision of the fungi classified as Gloeosporium. Bibl. Mycol. 24, 1-203.

Azevedo AR. 1976. A defesa sanitaria da oliveira em Portugal. Instituto Nacional de Investigação Agrária, Oeiras, Portugal.

Ballio A, Bottalico A, Bounocore V, Carilli A, Di Vittorio V, Graniti A. 1969. Production and isolation of aspergillomarasmin B (lycomarasmic acid) from cultures of Colletotrichum gloeosporioides (Gloeosporium olivarum). Phytopathol. Mediterr. 8, 187-196.

Barranco D, Cimato A, Fiorino P, Rallo L, Touzani A, Castañeda C, Serafín E, Trujillo I. 2000. World catalogue of olive varieties. International Olive Oil Council y MundiPrensa, Madrid.

Bartolini G, Cerreti S. 2013. Olive germplasm (Olea europaea L.). http://www.oleadb.it. Consultado (26/04/2013).

Besnard G, Khadari B, Navascués M, Fernández-Mazuecos M, El Bakkali A, Arrigo N, Baali-Cherif D, Bronzini de Caraffa V, Santoni S, Vargas P, Savolainen V. 2013. The complex history of the olive tree: from Late Quaternary diversification of Mediterranean lineages to primary domestication in the northern Levant. Proc. R. Soc. B. http://dx.doi.org/10.1098/ rspb.2012.2833.

Biraghi A. 1934. Variazioni in ceppi di Gloeosporium olivarum Alm. di provenienze diverse. Boll. R. Staz. Patol. Veg. 14, 223-253.

Bompeix G, Julio EVR, Phillips DH. 1988. Glomerella cingulata (Stoneman) Spaulding et V. Schrenk, in Smith IM, Dunez J, Lelliot RA, Phillips DH, Archer SA, (Eds.) European handbook of plant diseases. Blackwell Scientic Pubblications, Oxford, England.

Borkow G, Gabbay J. 2009. Copper, an ancient remedy returning to fight microbial, fungal and viral infections. Curr. Chem. Biol. 3, 272-278. http://dx.doi.org/10.2174/187231309789054887.

Bottalico A. 1973. Qualche dato sperimentale sugli effetti fitotossici dell'aspergillomarasmina B associata a vari ioni metallici. Phytopathol. Medit. 12, 1-6.

Bousquets JF, Vegh I, Pouteau-Thouvenot M, Barbier M. 1971. Isolement de l'aspergillomarasmine A de cultures de Colletotrichum gloeosporioides, agent pathogène de saules. Ann. Phytopathol. 3, 407-408.

Branz-Sobreiro J. 1992. Guia para a protecçao fitosanitaria da oliveira. Centro nacional de proteç̧ao de produçao agrícola, Lisboa, Portugal.

Bronzini de Caraffa V, Maury J, Gambotti C, Breton C, Bervillé A, Giannettini J. 2002. Mitochondrial DNA variation and RAPD mark oleasters, olive and feral olive from Western and Eastern Mediterranean. Theor. Appl. Genet. 104, 1209-1216. http://dx.doi.org/10.1007/ s00122-002-0883-7.

Bū'l-Jayr (siglo XI). Kitab Al-Filãha. Tratado de Agricultura. Trad. J.M. Carabaza (1991), AECI, Madrid.

Caballero JM, del Río C, Barranco D, Trujillo I. 2006. The olive world germplasm bank of Córdoba, Spain. Olea 25, 14-19.

Cacciola SO, Faedda R, Sinatra F, Agosteo GE, Schena S, Frisullo S, Magnano di San Lio G. 2012. Olive Anthracnose. J. Plant Pathol. 94, 29-44.

Cacciola SO, Pane A, Agosteo GE, Magnano di San Lio G. 1996. Osservazioni sull' epidemiologia dell'anthracnosi dell'olivo in Calabria. Inform. Fitopatol. 6, 27-32.

Carvalho MT, Simoe-Lopes P, Monteiro da Silava MJ. 2008. Influence of different olive infection rates of Colletotrichum acutatum on some important olive oil chemical parameters. Acta Hort. 791, 555-559.

Ciccarone A. 1950. Considerazioni biologiche e sistematiche sull'agente della «lebbra» delle olive recenttemente osservata nel Leccese. Boll. Staz. Pat. Veg. Roma 5, 143-165.

Council Regulation (EEC). 2007. No. 834/2007 of 28 of June 2007. On organic production and labelling of organic products and repealing regulation. Off. J. Eur. Commun. L189, 1-23.

Council Regulation (EEC). 1991. No. 2568/91 of $11^{\text {th }}$ of July 1991. On the characteristics of olive oil and olive-residue oil and on the relevant methods of analysis. Off. J. Eur. Commun. L248, 1-83.

Coutinho MP. 1968. Algumas notas sobre a gafa da azeitona. Ao Seviço da Lavoura $\mathbf{8 5}, 1-7$

Damm U, Cannon PF, Woudenberg JHC, Crous PW. 2012. The Colletotrichum acutatum species complex. Stud. Mycol. 73, 37-113. http://dx.doi.org/10.3114/sim0010.

Eurostat. 2007. The use of plant protection products in the European Union: data 1992-2003. Statistical book. European Communities, Luxembourg.

Faedda R, Agosteo GE, Schena L, Mosca S, Frisullo S, Magnano di San Lio G, Cacciola SO. 2011. Colletotrichum clavatum sp. nov. identified as the causal agent of olive Anthracnose in Italy. Phytopathol. Medit. 50, 283-302.

Famiani F, Proietti P, Farineli D, Tombesi A. 2002. Oil quality in relation to olive ripening. Acta Hort. 586, 671-674.

FAO. 2012. The Food and Agriculture Organization of the United Nations Statistical Database (FAOSTAT). http:// faostat.fao.org. Consultado (26/04/2013).

Farr DF, Rossman AY. 2013. Fungal Databases, Systematic Mycology and Microbiology Laboratory, ARS, USDA. http://nt.ars-grin.gov/fungaldatabases/. Consultado (20/11/ 2013)

Fernández MV. 1973. Introducción a la Fitopatología. $3^{\mathrm{a}} \mathrm{Ed}$ Vol. III. Hongos. Colección Científica del INTA, Buenos Aires. Argentina

García F, Pedret E, Marco V, Duatis JJ. 1997b. Sensibilidad de diversas variedades de olivo al hongo Colletotrichum gloeosporioides. Frut. Profesional 88, 60-63.

García F. 1995. Micoflora asociada a la aceituna. Su repercusión en la calidad del aceite. Agricultura 760, 931-933.

García F. 1998. Micosis de las aceitunas y su incidencia en la calidad del aceite. Phytoma España 102, 171-175.

García F. Duatis JJ, Marco V, Pedret E. 1997a. Influencia de los ataques fúngicos en la pérdida de calidad del aceite de oliva. Frut. Profesional 88, 131-135.

Gomes S, Prieto P, Martins-Lopes P, Carvalho T, Martin A, Guedes-Pinto H. 2009. Development of Colletotrichum acutatum on tolerant and susceptible Olea europaea L. cultivars: a microscopic analysis. Mycopathol. 168, 203-211. http://dx.doi.org/10.1007/s11046-009-9211-y.

Gómez-Caravaca AM, Cerretani L, Bendini A, Segura-Carretero A, Fernández-Gutiérrez A, Del Carlo M, Compagnone D, Cichelli A. 2008. Effects of fly attack (Bactrocera oleae) on the phenolic profile and selected chemical parameters of olive oil. J. Agr. Food Chem. 56, 4577-4583. http://dx.doi. org/10.1021/jf800118t.

Gómez-Coca RB, Moreda W, Pérez-Camino MC. 2012. Fatty acid alkyl esters presence in olive oil vs. organoleptic assessment. Food Chem. 135, 1205-1209. http://dx.doi. org/10.1016/j.foodchem.2012.05.053. 
González-Fragoso, R. 1914. Aclaraciones a los hongos conocidos con el nombre de "Repilos". Bol. Soc. Esp. Hist. Nat. 14, 291-293.

Gorter GJMA. 1956. Antracnose fungi of olives. Nature 178, 1129-1130. http://dx.doi.org/10.1038/1781129a0.

Graniti A, Frisullo S, Penissi A, Magnano L. 1993. Infections of Glomerella cingulata on olive in Italy. EPPO Bull. 23, 457465. http://dx.doi.org/10.1111/j.1365-2338.1993.tb01353.x.

Graniti A, Laviola C. 1981. Sguardo generale alle malattie parassitarie dell'olivo. Informatore Fitopatol. 31, 77-92.

Graniti A. 1953. La "lebbra delle" Olive in Sicilia. Notiz. Malatt. Piante 27, 27-32.

Graniti A. 1954. La "lebbra delle" Olive in Sicilia. Olivicoltura 9, $1-5$

Green PS. 2002. A revision of Olea L. (Oleaceae). Kew Bulletin 57, 91-140. http://dx.doi.org/10.2307/4110824.

Guerfel M, Ben Mansour M, Ouni Y, Guido F, Boujnah D, Zarrouk M. 2012. Triacylglycerols composition and volatile compounds of virgin olive oil from cv. Chemlali: Comparison among different planting densities. Sci. World J. 2012, 354019. http://dx.doi.org/10.1021/jf102724f.

Guerfel M, Zaghdoud C, Jebahi K, Boujnah D, Zarrouk M. 2010. Effects of the planting density on virgin olive oil quality of 'Chemlali' olive trees (Olea europaea L.). J. Agric. Food Chem. 58, 12469-12472. http://dx.doi.org/10.1021/ jf102724f.

Hemmi T, Kurata S. 1935. Contributions to the knowledge of Anthracnose of plants II, on Gloeosporium olivarum Alm. causing the olive Anthracnose. J. Soc. Trop. Agric. Taiwan 6, 573-583

Hibbett DS, Binder M, Bischoff JF, Blackwell M, Cannon PF, Eriksson OE, Huhndorf S, James T, Kirk PM, Lücking R, Lumbsch T, Lutzoni F, Matheny PB, Mclaughlin DJ, Powell MJ, Redhead S, Schoch CL, Spatafora JW, Stalpers JA, Vilgalys R, Aime MC, Aptroot A, Bauer R, Begerow D, Benny GL, Castlebury LA, Crous PW, Dai YC, Gams W, Geiser DM, Griffith GW, Gueidan C, Hawksworth DL, Hestmark G, Hosaka K. Humber RA, Hyde K, Ironside JE, Kõljalg U, Kurtzman CP, Larsson KH, Lichtwardt R, Longcore J, Miadlikowska J, Miller A, Moncalvo JM, Mozley-Standridge S, Oberwinkler F, Parmasto E, Reeb V, Rogers JD, Roux C, Ryvarden L, Sampaio JP, Schüßler A, Sugiyama J, Thorn RG, Tibell L, Untereiner WA, Walker C, Wang Z, Weir A, Weiß M, White MM, Winka K, Yao YJ, Zhang N. 2007. A higher-level phylogenetic classification of the Fungi. Mycol. Res. 111, 509-547. http://dx.doi.org/10.1016/j.mycres.2007.03.004

Iannotta I, Perri E, Siriani R, Tocci C. 1999. Influence of Colletotrichum gloeosporioides (Penzing) and Camarosporium dalmatica (Thum) attacks on olive oil quality. Acta Hort. 474, 399-401.

Inglese P, Famiani F, Galvano F, Servili M, Esposto S, Urbani S. 2011. Factors affecting extra virgin olive oil composition. Hortic. Rev. 38, 83-147.

International Olive Oil Council. 2012. Norma Comercial aplicable a los aceites de oliva y los aceites de orujo de oliva. (COI/T.15/NC n 3/Rev. 2)

Keys A, Scardi V, Bergami G. 1952. The trends of serum cholesterol levels with age. Lancet 2, 209-210. http://dx.doi. org/10.1016/S0140-6736(52)91544-4.

Keys A. 1970. Coronary heart disease in seven countries. Circulation, 41 (Suppl. I).

Latinovic J, Vucinic Z. 2002. Cultural characteristics, pathogenicity, and host range of Colletotrichum gloeosporioides isolated from olive plants in Montenegro. Acta Horticul. 586, 753-755.

Loprieno N, Tenerini I. 1960. Indagini sul Gloeosporium olivarum Alm., agente della "lebbra" delle olive. Phytopathol. Z. 39, 262-290. http://dx.doi.org/10.1111/j.1439-0434.1960. tb01906.x.

Margarita L, Porta-Puglia A, Quacquarelli A. 1986. Colletotrichum acutatum, nuovo patogeno dell'olivo in Cina e confronto con l'agente della «lebbra» dell'olivo. Ann. Ist. Sper. Patol. Veg. Roma 11, 125-133.

Martelli GP, Piglionica V. 1961. Tre anni di lotta contra la Lebbra delle olive in Puglia. Phytopathol. Medit. 3, 101-112.
Martelli GP. 1959. La lebbra delle olive. Presenza e diffusione in Calabria. Italia Agric. 96, 905-914.

Martelli GP. 1960. Primo contributo alla conoscenza della biologia di Gloeosporium olivarum Alm. Phytopathol. Medit. 1, 31-43.

Martelli GP. 1961. Acervuli di Gloeosporium olivarum Alm. su foglie di olivo. Phytopathol. Medit. 1, 43-51.

Martín MP, García F. 1999. Colletotrichum acutatum y C. gloeosporioidescause anthracnose on olives. Eur J Plant Pathol. 105, 733-741. http://dx.doi.org/10.1023/A:1008785703330.

Martín MP, García-Figueres F, Trapero A, 2002. Iniciadores especificos para detectar las especies de Colletotrichum causantes de la antracnosis de los olivos. Bol. Sanl. Veg. Plag. 28, 43-50.

Matalas AL, Zampelas A, Staurnos V, Wolinski I. 2001. The Mediterranean Diet Constituents and Health Promotion. CRC Press, Boca Raton, USA

Mateo-Sagasta E. 1968. Estudios básicos sobre Gloeosporium olivarum Alm. (Deuteromiceto Melanconial). Bol. Patol. Veg. Entomol. Agric. 30, 31-135.

Mincione A, Valenzise M, Runcio A, Poiana M, Agosteo GE, Taccone PL. 2004. Ricerche sugli oli di oliva vergini calabresi. Influenza delle fitopatie sulle caratteristiche qualitative degli oli. Nota I-Effetti diretti degli attacchi di Antracnosi. Riv. It. Sost. Grasse 81, 9-17.

Montag J, Schreiber L, Schönherr J. 2006. An in vitro study of the nature of protective activities of copper sulphate, copper hydroxide and copper oxide against conidia of Venturia inaequalis. J. Phytopathol. 154, 474-481. http://dx.doi. org/10.1111/j.1439-0434.2006.01132.x.

Montironi RA. 1954. Olivo. Frantoio variedad resistente a la Antracnosis. Pulverizaciones recomendables en montes de olivo Idia 106, 176-177

Moral J, Alsalimiya M, Muñoz-Díez C, León L, de la Rosa R, Trapero A. 2006. Evaluación de preselecciones de olivo por su resistencia a Repilo y Antracnosis. Act. Hort. 45, $177-178$.

Moral J, Ávila A, López-Doncel LM, Alsalimiya M, Oliveira R, Gutiérrez F, Navarro N, Bouhmidi K, Benali A, Roca L, Trapero A. 2005. Resistencia a los Repilos de distintas variedades de olivo. Vida Rural 208, $34-40$

Moral J, Bouhmidi K, Trapero A, 2008. Influence of fruit maturity, cultivar susceptibility, and inoculation method on infection of olive fruit by Colletotrichum acutatum. Plant Dis. 92, 1421-1426. http://dx.doi.org/10.1094/ PDIS-92-10-1421

Moral J, Cherifi F, Muñoz-Díez C, Xavier CJ, Trapero Casas A. 2009a. Infection of olive seeds by Colletotrichum acutatum and its effect on germination. Phytopathology 99, S88.

Moral J, Jurado-Bello J, Sánchez MI, Oliveira R, Trapero A. 2012. Effect of temperature, wetness duration, and planting density on olive anthracnose caused by Colletotrichum spp. Phytopathology 102, 974-981. http://dx.doi.org/10.1094/ PHYTO-12-11-0343.

Moral J, Jurado-Bello J, Trapero A. 2011. Effect of temperature and relative humidity on mycelial growth, conidial germination and fruit infection by Colletotrichum spp. causing olive Anthracnose. IOBC/WPRS Bull. 79, 14.

Moral J, Oliveira R, Roca LF, Cabello D, Trapero A. 2009b. Control of olive Anthracnose caused by Colletotrichum spp. IOBC/WPRS Bull. 78, 55 .

Moral J, Oliveira R, Trapero A, 2009c. Elucidation of disease cycle of olive anthracnose caused by Colletotrichum acutatum. Phytopathology 99, 548-556. http://dx.doi.org/ 10.1094/PHYTO-99-5-0548.

Moral J Trapero A. 2009a. Assessing the susceptibility of olive cultivars to anthracnose caused by Colletotrichum acutatum. Plant Dis. 93, 1028-1036. http://dx.doi.org/10.1094/ PDIS-93-10-1028.

Moral J, Trapero A. 2009b. Resistencia del olivo a la Antracnosis causada por Colletotrichum spp. Boletín SEF 66, 22-30.

Moral J, Trapero A. 2010. Fuentes de inóculo y dinámica de la infección en la Antracnosis del olivo causada por Colletotrichum spp. XV Congreso SEF (Vitoria).

Moral J, Trapero A. 2012. Mummified fruit as a source of inoculum and disease dynamics of olive anthracnose caused 
by Colletotrichum spp. Phytopathology 102, 982-989. http://dx.doi.org/10.1094/PHYTO-12-11-0344.

Mraicha F, Ksantini M, Zouch O, Ayadi M, Sayadi S, Bouaziz M. 2010. Effect of olive fruit fly infestation on the quality of olive oil from Chemlali cultivar during ripening. Food Chem. Toxicol. 48, 3235-3241. http://dx.doi.org/10.1016/j. fct.2010.08.031.

Mugnai L, Surico G, Rogazzi A. 1993. Glomerella cingulata on olive in India: morphological and pathological notes. EPPO Bull. 23, 449-455. http://dx.doi. org/10.1111/j.1365-2338.1993.tb01352.x.

Oliveira R, Moral J, Bouhmidi K, Trapero A. 2005. Caracterización morfológica y cultural de aislados de Colletotrichum spp. causantes de la antracnosis del olivo. Bol. San. Veg. Plagas 31, 531-548.

Pennisi M, Agosteo GE, Grasco S. 1993. Chemical control of the olive rot caused by Glomerella cingulata. EPPO Bull. 23, 467472. http://dx.doi.org/10.1111/j.1365-2338.1993.tb01354.x.

Pérez M. 2011. Evaluación en campo de fungicidas, sales de calcio y extractos vegetales para el control de la antracnosis del olivo causada por Colletotrichum spp. Trabajo Profesional Fin de Máster, ETSIAM, Córdoba.

Pérez-Camino MC, Moreda W, Mateos R, Cert A. 2002. Determination of esters of fatty acids with low molecular weight alcohols in olive oils. J. Agric. Food Chem. 50, 47214725. http://dx.doi.org/10.1021/jf025542+.

Pontis RE, Hansen HN. 1942. Olive Anthracnose in the United States. Phytopathology 32, 642-644.

Prota U. 1995. Le malattie dell'olivo. Infor. Fitopatol. 45, 16-26.

Prusky D, Freeman S, Dickman MB. 2000. Colletotrichum: Host Specificity, Pathology, and Host-Pathogen Interaction. The American Phtopathological Society. St. Paul MN, USA

Rahman M. Punja ZK. 2007. Calcium and plant disease in Datnoff LE, Elmer WH, Huber DM (Eds) Mineral nutrition and plant disease. APS Press, San Paul, MN, 79-93.

Rallo, L. 2005. Variedades del olivo en España: una aproximación cronológica. in Rallo L, Barranco D, Caballero JM del Río C, Martín A, Tous J, Trujillo I (Eds) Variedades del olivo en España. Junta de Andalucía, MAPA y Ediciones Mundi-Prensa, Madrid, 15-44.

Rhouma A, Triki MA, Msallem M. 2010. First report of olive anthracnose caused by Colletotrichum gloeosporioides in Tunisia. Phytopathol. Med. 49, 95-98.

Roca LF, Moral J, Viruega JR, Ávila A, Oliveira R, Trapero A. 2007. Copper fungicides in the control of olive diseases. Olea 26, 48-50.

Runcio A, Sorgonà L, Mincione A, Santacaterina S, Poiana M. 2008. Volatile compounds of virgin olive oil obtained from Italian Cultivars grown in Calabria. Effects of processing methods, cultivar, stone removal, and antracnose attack. Food Chemistry 106, 735-740. http://dx.doi.org/10.1016/j. foodchem.2007.06.051

Saponaro A. 1953. Presenza di Gloeosporium olivarum Alm. sugli organi vegetativi dell'olivo nel Leccese e nel Brindisino. Ann. Sperim. Agraria 7, 609-619.

Schena L, Mosca S, Cacciola SO, Faedda R, Sanzani SM, Agosteoa GE, Sergeeva V, Magnano di San Lioa G. 2013. Species of the Colletotrichum gloeosporioides and C. boninense complexes associated with olive anthracnose. Plant Pathol. http://dx.doi.org/10.1111/ppa.12110.

Segura R. 2003. Evaluación de microorganismos antagonistas para el control biológico del Repilo y la Antracnosis del olivo. Tesis doctoral, ETSIAM, Córdoba.

Sergeeva V, Nair NG, Spooner-Hart R. 2008a. Evidence of early flower infection in olives (Olea europaea) by Colletotrichum acutatum and C. gloeosporioides causing anthracnose disease. Australas. Plant Dis. Not. 3, 81-82. http://dx.doi. org/10.1071/DN08032.
Sergeeva V, Spooner-Hart R, Nair NG. 2008b. First report of Colletotrichum acutatum and C. gloeosporioides causing leaf spots of olives (Olea europaea) in Australia. Australas. Plant Dis. Not. 3, 143-144. http://dx.doi.org/10.1071/ DN08055.

Sergeeva V. 2011. Disease resistance and adaptability of olive cultivars. Australas. N. Zealand Olivegro. Proces. 81, 27-29.

Sharma RL, Kaul JL. 1990. Field evaluation of fungicide for control of olive Anthracnose. Indian J. Mycol. Plant Pathol. 20, 185-187.

Sutton BC. 1980. The Coelomycetes. Fungi imperfecti with pycnidia, acervula and stromata. Commonwealth Mycological Institute, Kew, England.

Talhinhas P, Neves-Martins J, Oliveira H, Sreenivasaprasad S. 2009. The distinctive population structure of Colletotrichum species associated with olive anthracnose in the Algarve region of Portugal reflects a host-pathogen diversity hot spot. FEMS Microb. Lett. 296, 31-38. http://dx.doi. org/10.1111/j.1574-6968.2009.01613.x.

Talhinhas P, Sreenivasaprasad S, Neves-Martins J, Oliveira H. 2005. Molecular and phenotypic analyses reveal association of diverse Colletotrichum acutatum groups and a low level of $C$. gloeosporioides with olive anthracnose. Appl. Environ. Microbiol. 71, 2987-2998. http://dx.doi. org/10.1128/AEM.71.6.2987-2998.2005.

Tamendjari A, Angerosa F, Mettouchi S, Bellal MM. 2009. The effect of fly attack (Bactrocera oleae) on the quality and phenolic content of Chemlal olive oil. Grasas Aceites $\mathbf{6 0}$, 507-513. http://dx.doi.org/10.3989/gya.032209

Trapero A, Blanco MA. 2008. Enfermedades. In Barranco D, Fernández-Escobar R, Rallo L (Eds) El cultivo de olivo. Coedición Junta de Andalucía/Mundi-Prensa, Madrid, 595-656.

Trapero A, Roca LF, Moral J. 2009. Perspectivas futuras del control químico de las enfermedades del olivo. Phytoma España 212, 80-82.

Uceda M, Hermoso M, Aguilera MP. 2008. La calidad del aceite de oliva. In Barranco D, Fernández-Escobar R, Rallo L (Eds) El cultivo de olivo. Coedición Junta de Andalucíal Mundi-Prensa, Madrid, 699-727.

Vichi S, Pizzale L, Conte LS, Buxaderas S, Lopez-Tamames E. 2003. Solid-phase Microextraction in the analysis of virgin olive oil volatile fraction: Modifications induced by oxidation and suitable markers of oxidative status. $J$. Agr. Food Chem. 51, 6564-6571. http://dx.doi.org/10.1021/ jf030268k.

Vucinic Z, Latinovic J, Metzidakis IT, Voyiatzis DG. 1999. Colletotrichum gloeosporioides, a new olive (Olea europaea L.) parasite in Yugoslavia. Acta Hortic. 474, 577-579.

Weir BS, Johnston PR, Damm U, 2012. The Colletotrichum gloeosporioides species complex. St. in Mycol. 73, 115-80. http://dx.doi.org/10.3114/sim0011.

Xaviér CJ, Moral J, Cherefii F, Díez CM, Trapero A. 2012. Resistencia del acebuche a la Antracnosis del olivo causada por Colletotrichum spp. y su posible origen. XVI Congreso SEF (Málaga).

Xaviér CJ, Moral J, Trapero 2010. Caracterización de la virulencia de Colletotrichum acutatum, agente de la Antracnosis del olivo. XV Congreso SEF (Vitoria).

Zachos DG, Makris SA. 1963. Recherches sur le Gloeosporium olivarum en Grèce. II. Symptomatologie de la maladie. III. Epidémiologie de la maladie. Ann. Inst. Phytopathol. Benaki, 5, 128-130

Zohary D, Spiegel-Roy P. 1975. Beginning of fruit growing in the old world. Science 187, 319-327. http://dx.doi.org/10.1126/ science.187.4174.319

Zohary D. 1994. The wild genetic resources of the cultivated olive. Acta Hort., 356, 62-65. 